\title{
Vortical flow structure identification and flow transport in arteries
}

\author{
D J Doorly, S J Sherwin, P.T. Franke and J. Peiró \\ Aeronautics Dept., Imperial College London
}

\section{Introduction}

Our interest in vortices arises for two reasons. Firstly, at moderate to large Reynolds numbers (at least 100) which characterise flow in larger arteries, vortices are quite persistent. The presence of vortices in a flow may exert a strong influence on its behaviour, although tracking vortices may be difficult as they can evolve rapidly. The effects of vortices or vortical structures are particularly evident when considering both flow stability, and the processes of mixing and transport by the flow. The object of this paper is to examine the dynamics both of vortex motion and of particle transport in arteries, and to relate these to parameters such as geometry and unsteadiness. The Lagrangian particle tracking and the vortex dynamic techniques which are described should help in understanding arterial fluid dynamics and suggest new approaches to modelling.

To begin, we could ask what does the word vortex mean? A vortex describes a circulating region of a flow, and for most people the term implies the fluid is rotating in some way. Familiar examples include the flow down a bath plug hole, tornados, smoke rings, the flow behind the tips of an aircraft wing in flight, and the whirlpool eddies in the wake of a bridge pier set in a rapid stream. It appears odd to admit that fluid dynamicists have difficulties in defining precisely what constitutes a vortex, and that when pressed many prefer to discuss vortical motions, or vortical structures rather than to talk of vortices. Despite this vagueness, one often finds that discussions of vortical motions are accompanied by rotating arm or hand gestures, which appear involuntarily. At a basic level we clearly associate the word vortex with the presence of rotation of elements of the flow. 
Computer Methods in Biomechanics and Biomechanical Engineering, 2002 Vol.5 (3) pp. 261-275

The difficulty with characterising a vortex in terms of rotation about some point or axis is that in complex flows vortices can be highly distorted, split or merge over quite short distances, and it may be impossible to identify any point or axis about which particles complete a full rotation. Interest in the dynamics of turbulent flows has recently led to new measures to identify vortical structures. These seem to offer some assistance in identifying vortices in general flows and we describe them in the context of large arterial flows. In the following sections, vorticity is first reviewed briefly and an existing vortical structure identification measure is discussed. A bypass graft flow configuration illustrates the application of vortex dynamics and structure identification to a complex flow which may be steady or unsteady. Finally other aspects of the computational modelling of transport and mixing of particles by the flow in arteries are discussed.

\section{Velocity gradient, vorticity, vortex identification and vortex dynamics}

\subsection{Velocity gradient and vorticity}

Consider the flow in the vicinity of a point $P$ which is fixed in space with position vector $\boldsymbol{x}_{\boldsymbol{P}}$. (The instantaneous position of a point i.e. the Lagrangian co-ordinate is denoted by $\boldsymbol{X}_{\boldsymbol{P}}$ ). The velocity near $P$ can be written as

$$
\begin{aligned}
\boldsymbol{u}\left(\boldsymbol{x}_{P}+\boldsymbol{d} \boldsymbol{x}\right) & =\boldsymbol{u}\left(\boldsymbol{x}_{P}\right)+\left.\boldsymbol{d} \boldsymbol{x} \cdot \nabla \boldsymbol{u}\right|_{P}+O\left(d x^{2}\right) \\
& =\boldsymbol{u}\left(\boldsymbol{x}_{P}\right)+\boldsymbol{d} \boldsymbol{x} \cdot(\boldsymbol{S}+\boldsymbol{\Omega})+O\left(d x^{2}\right)
\end{aligned}
$$

where the velocity gradient tensor

$$
(\nabla u)_{i j}=\frac{\partial u_{j}}{\partial x_{i}}=u_{j, i}
$$

can be decomposed into symmetric, $\boldsymbol{S}$, and anti-symmetric, $\boldsymbol{\Omega}$, parts as

$$
S=\frac{1}{2}\left[\nabla \boldsymbol{u}+(\nabla \boldsymbol{u})^{T}\right] ; \quad \boldsymbol{\Omega}=\frac{1}{2}\left[\nabla \boldsymbol{u}-(\nabla \boldsymbol{u})^{T}\right] .
$$

$S$ represents stretching and $\boldsymbol{\Omega}$ rotation, and only three of the components $\Omega_{i j}$ are independent. Thus $\boldsymbol{\Omega}$ can be written as the outer product of the identity tensor with a vector; whilst examining $\Omega$ shows the appropriate vector is half the vorticity w. Thus: 


$$
\boldsymbol{\Omega}=-\frac{1}{2}(\boldsymbol{I} \wedge \boldsymbol{\omega}) ; \boldsymbol{\omega}=\nabla \wedge \boldsymbol{u}
$$

a)

so the components are related by

$$
\Omega_{i j}=\frac{1}{2}\left(u_{j, i}-u_{i, j}\right)=\frac{1}{2} \varepsilon_{i j k} \omega_{k} \quad ; \quad \omega_{k}=\varepsilon_{k i j} \Omega_{i j}
$$

b)

Taking the curl of the Navier Stokes equations eliminates pressure from the flow specification, and yields the vorticity transport equation:

$$
\frac{d \boldsymbol{\omega}}{d t}=\boldsymbol{\omega} \cdot \nabla \boldsymbol{u}+\nu \nabla^{2} \boldsymbol{\omega}
$$

where $d / d t$ represents the material derivative $\partial / \partial t+\boldsymbol{u} \cdot \nabla$. From this it follows that vorticity cannot be created within a flow, but can only enter by convection or diffusion across the boundaries. Decomposing the gradient $\nabla \boldsymbol{u}$ as $S+\Omega$, taking the right outer product of half the identity tensor $\left({ }^{1} / 2 \boldsymbol{I} \wedge\right)$ with both sides of equation $(2.5)$ and using the relations $\boldsymbol{S}=\boldsymbol{S}^{\boldsymbol{T}}, \boldsymbol{\Omega}=-\boldsymbol{\Omega}^{T}$ yields, after simplification the equivalent

$$
\frac{d \boldsymbol{\Omega}}{d t}=-(\boldsymbol{S} \boldsymbol{\Omega}+\boldsymbol{\Omega} \boldsymbol{S})+\nu \nabla^{2} \boldsymbol{\Omega}
$$

relation for the transport of $\Omega$ :

The existence of a vortex implies the presence of vorticity somewhere in the flow, even if it is only concentrated at a singular point. Vorticity does not imply a vortex however, as can be seen from decomposing the velocity gradient for a simple shear flow $\boldsymbol{u}=(j y, 0,0)$ above a plane wall $y=0$. This has constant vorticity with value $\dot{\gamma}$, but nowhere is there any complete rotation of a flow element. Instead a rectangular element is partly rotated $1 / 2 \gamma$ clockwise through the operator $\boldsymbol{\Omega}$, and undergoes a volume preserving stretch through $S$ which reduces the initial right angle of the element by ${ }^{1} / 2 \dot{\gamma}$. Physically the effect of the wall can be modelled as a negative reflection of the shear layer vorticity about the boundary to enforce the nothroughflow condition. 
Computer Methods in Biomechanics and Biomechanical Engineering, 2002 Vol.5 (3) pp. 261-275

For an identifiable vortex, some local concentration of vorticity away from the wall is needed, but the use of isovorticity surfaces for identification is fraught with difficulty, as discussed by Jeong \& Hussain (1995). Instead, two approaches that we found useful are:

(i) identification of a vortex from defined characteristics of the flowfield, and

(ii) qualitative modelling of the emergence of vortex structures from the vorticity.

\subsection{Vortex identification from flow characteristics.}

Jeong and Hussain (1995) propose that a vortex corresponds to a region where two eigenvalues of the symmetric part of the square of the velocity gradient are negative. The eigenvalues $\lambda_{1}, \lambda_{2}, \lambda_{3}$, of $\boldsymbol{S}^{2}+\boldsymbol{\Omega}^{2}$ are real, and labelling these in order $\lambda_{1} \geq \lambda_{2} \geq \lambda_{3}$, the condition is that:

$$
\lambda_{2}<0
$$

For a local pressure minimum in a plane, the Hessian $\left[p_{, i j}\right]$ of pressure, obtained by taking the gradient of the Navier-Stokes equations,

$$
-\frac{1}{\rho} \nabla(\nabla p)=\frac{d \boldsymbol{S}}{d t}+(\boldsymbol{S} \boldsymbol{S}+\boldsymbol{\Omega} \boldsymbol{\Omega})+\frac{d \boldsymbol{\Omega}}{d t}+(\boldsymbol{S} \boldsymbol{\Omega}+\boldsymbol{\Omega} \boldsymbol{S})-\nu \nabla^{2}(\boldsymbol{S}+\boldsymbol{\Omega})
$$

should have two positive eigenvalues. Jeong \& Hussain (1995) argue for the removal of both the unsteady strain term, $d \boldsymbol{S} / d t$, which can also produce a local pressure minimum, and the viscous terms, which can mask the pressure minimum. Removing also the vorticity transport equation (2.6) from (2.8) leaves only $\boldsymbol{S}^{2}+\boldsymbol{\Omega}^{2}$, and hence the condition (2.7).

A more direct argument for (2.7) in our view follows from considering the relative acceleration about a point. Let $\boldsymbol{d} \boldsymbol{x}$ represent the vector from $P$ to a nearby point $Q$ at time $t$, let $\boldsymbol{d} \boldsymbol{X}$ represent its initial separation from $P$, and let $\boldsymbol{F}$ be the deformation gradient $\partial x_{i} / \partial X_{j}$. Then expanding the relative position and velocity fields about $P$ from (2.1), to first order:

$$
\begin{gathered}
d \boldsymbol{x} \simeq \boldsymbol{F} \cdot d \boldsymbol{X} \\
\frac{d(d \boldsymbol{x})}{d t} \equiv d \dot{\boldsymbol{x}} \simeq(\nabla \boldsymbol{u})^{T} \cdot d \boldsymbol{x}=d \boldsymbol{x} \cdot \nabla \boldsymbol{u}
\end{gathered}
$$


since

$$
\frac{d}{d t}\left(d x_{i}\right) \simeq \frac{\partial u_{i}}{\partial X_{j}} \cdot d X_{j}=\frac{\partial u_{i}}{\partial x_{k}} \frac{\partial x_{k}}{\partial X_{j}} d X_{j}=[\nabla u]_{i j}^{T} d x_{j}
$$

Hence

$$
d \ddot{\boldsymbol{x}} \simeq d \dot{\boldsymbol{x}} \cdot \nabla \boldsymbol{u}+d \boldsymbol{x} \cdot \frac{d}{d t}(\nabla \boldsymbol{u})
$$

A vortical structure which exists in the flowfield in a neighbourhood of point $P$ at any given time, and persists thereafter for any finite time interval should be identifiable from the relative motion of $Q$ about $P$, neglecting instantaneous distortions produced by local acceleration. Omitting temporal or convective changes in the velocity gradient (unsteady strain and transport of vorticity) when considering the acceleration of $Q$ with respect to $P$ implies that (2.10a) reduces to

$$
\boldsymbol{d} \ddot{\boldsymbol{x}} \simeq \boldsymbol{d} \tilde{\ddot{\boldsymbol{x}}} \equiv \boldsymbol{d} \dot{\boldsymbol{x}} \cdot \nabla \boldsymbol{u}=(\nabla \boldsymbol{u})^{T}(\nabla \boldsymbol{u})^{T} \cdot d \boldsymbol{x}
$$

For the relative motion of $Q$ to correspond to circular or spiral pathlines about $P$ in a plane at the given time, the radial component of acceleration must be negative for any orientation of the vector from $P$ to $Q$ in the plane. But

$$
d \boldsymbol{x} \cdot d \tilde{\ddot{\boldsymbol{x}}}=d \boldsymbol{x} \cdot\left((\nabla \boldsymbol{u})^{T}(\nabla \boldsymbol{u})^{T} \cdot d \boldsymbol{x}\right)=\left(\boldsymbol{S}^{2}+\boldsymbol{\Omega}^{2}\right): d \boldsymbol{x} d \boldsymbol{x}
$$

since the inner product of $(\nabla \boldsymbol{u})^{T}(\nabla \boldsymbol{u})^{T}$ with $\boldsymbol{d x} \boldsymbol{d x}$ (the tensor product $\boldsymbol{d x} \otimes \boldsymbol{d x}$ ) removes the anti-symmetric terms. Hence (2.7) follows from (2.11) purely from considerations of the local relative motion of point pairs; equally however, inward radial acceleration implies a pressure minimum.

\section{$\underline{2.3}$ Vortex dynamics}

The velocity field in incompressible flow can be decomposed as the sum of an irrotational component derived from a scalar potential and a rotational component $\boldsymbol{u}_{\mathbf{v}}$,

$$
\boldsymbol{u}=\nabla \varphi+\boldsymbol{u}_{\mathbf{v}}=\nabla \varphi+\nabla \wedge \boldsymbol{a} ; \text { where } \nabla^{2} \boldsymbol{a}=-\boldsymbol{\omega}
$$


The potential component is found by applying the inviscid no-throughflow condition, and $\boldsymbol{u}$ is then determined from $\boldsymbol{\omega}$. The force $\boldsymbol{f}$ on an element of wall surface with normal $\boldsymbol{n}$ is:

$$
f=\boldsymbol{n} \cdot\left(-p \boldsymbol{I}+\mu \nabla \boldsymbol{u}+\mu(\nabla \boldsymbol{u})^{T}\right)=-p \boldsymbol{n}+\mu(2 \boldsymbol{n} \cdot \nabla \boldsymbol{u}-\boldsymbol{n} \cdot 2 \Omega)=-p \boldsymbol{n}+\mu \boldsymbol{\omega} \wedge \boldsymbol{n}
$$

where $\boldsymbol{n} \cdot \nabla \boldsymbol{u}=0$ because of no-slip and incompressibility. Thus the tangential force or skin friction vector is given by $\boldsymbol{\omega} \wedge \boldsymbol{n}$, which integrated over the wall must be balanced by the pressure drop in the flow direction. So the pressure gradient is linked to the flux of vorticity from the wall, which in turn depends on the imposed boundary vorticity $\omega$ (wall).

Unless separation occurs, the velocity is approximately parallel to the surface throughout the near wall region. The initial transport of vorticity into the flow interior then occurs only by diffusion, spreading to a distance of order $(\nu t)^{1 / 2}$. For attached flow at high Reynolds number $(R e)$, changes in wall vorticity have little immediate effect on the interior field, and internal vortical structures evolve over considerable distances with little modification by the boundary vorticity. Viscous effects on the interior field are likewise gradual at high $R e$, so the vorticity transport equation (2.5) may be modelled inviscidly over a local region.

Consider an element $\boldsymbol{d} \boldsymbol{x}_{P}$ of a line which moves with the flow (a material line element), and assume that it is aligned with the local vorticity vector at some instant,

$$
d x_{P}=\varepsilon \omega_{P}
$$

Then (2.1) and, omitting viscous terms, (2.5) imply

$$
\frac{d\left(\boldsymbol{d} \boldsymbol{x}_{\boldsymbol{P}}-\varepsilon \boldsymbol{\omega}_{P}\right)}{d t}=\left(\boldsymbol{d} \boldsymbol{x}_{\boldsymbol{P}}-\varepsilon \boldsymbol{\omega}_{P}\right) \cdot \nabla \boldsymbol{u}=0
$$

So neglecting viscosity, a vortex line is convected with the fluid (Helmholtz second law). Replacing the element of a material line by an element of a tube of vortex lines with cross-section $\delta A_{p}$, mass conservation and (2.13) imply $\omega_{p} \delta A_{p}$ is constant. Since 
incompressibility requires the volume $\left|\boldsymbol{d} \boldsymbol{x}_{P}\right| \delta A_{p}$ remains constant, $\delta A_{p} \propto 1 /\left|\boldsymbol{d} \boldsymbol{x}_{P}\right|$, and stretching of the flow implies an increase in vorticity.

From the Helmholtz relation (2.14) above, we can obtain a qualitative physical model for the evolution of the flow vorticity and the generation of vortex structures by tracking material lines aligned with the vorticity vector, over distances sufficiently short for viscous dissipation and boundary vorticity flux to be neglected. Using two marker rings, one nearer the axis, and the other nearer the wall highlights differences in vorticity evolution at different locations, and is indicative of some aspects of the mixing.

\section{Vortical structures in graft flows}

\section{$\underline{3.1 \text { Vorticity in steady pipe flow }}$}

For steady flow in a circular pipe the vorticity (in cylindrical coordinates $\boldsymbol{\omega}=\left(0, \omega_{\theta}, 0\right)$ ) varies linearly with radius. Over a length $d x$ it can be discretised as a series of vortex rings, (fig. 1), whose circulation decreases with ring radius. A single ring with circulation:

$$
\Gamma_{0}=\int \omega d r \cdot d x=-2 \bar{u} d x
$$

may be used as a coarse approximation, with a radius of either $1 / \sqrt{2}$ times that of the pipe to match the impulse (Saffman, 1992) of the vorticity distribution, or $(2 / 5)^{1 / 3}$ of the pipe radius to match the angular impulse. It is thus reasonable to choose a radius $3 / 4$ that of the pipe for a ring of marker particles designed to track the evolution of the mean vorticity. Clearly the evolution of a single material ring will only capture part of the dynamics, and it must be emphasised that this approach is distinctly qualitative. To visualise the core flow behaviour, we have also used a ring with a radius $1 / 4$ that of the pipe.

It has been brought to our attention that Agrawal et al (1978) also discussed the distortion of the vorticity distribution for flow entering a bend to explain the initial inviscid secondary core flow and the subsequent fully developed Dean flow structure. 
Although they did not seek an appropriate location to model the mean vorticity in fully developed flow, their application of essentially inviscid dynamics to the rotational entrance flow precedes our approach, and suggests a comparative study of the entrance flow problem.

\subsection{Graft model geometries and solution of the flowfield}

We consider firstly the problem of flow in an end-to-side anastomosis, where the host vessel is assumed to be fully occluded upstream of the junction. Bypass graft flows have been the subject of many computational and experimental investigations including Hofer et al (1996) and White et al (1993). Detailed computational and experimental results exist for the model geometry of fig. 2, which shows a 45 degree anastomosis (or junction) of equi-diameter cylindrical model graft and artery, e.g. Steinman et al (1996), Ethier et al (2000). Computational results for this and for a modified 'non-planar' configuration, (where the portion of the graft just upstream of the anastomosis comprises a quarter section of a torus), are documented for both steady flow (Sherwin et al 2000) and for unsteady flow (Papaharilaou et al 2001).

The spectral $h / p$ finite element procedure is used to solve for the flow for most of the results presented and is described in the latter two references. In essence however, the flow equations

$$
\frac{\partial \boldsymbol{u}}{\partial t}=-\nabla p+v \boldsymbol{L}(\boldsymbol{u})+N(\boldsymbol{u})
$$

where

$$
\boldsymbol{L}(\boldsymbol{u}) \equiv \nabla^{2} \boldsymbol{u} \quad ; \quad \boldsymbol{N}(\boldsymbol{u}) \equiv \boldsymbol{u} \cdot \nabla \boldsymbol{u}=\frac{1}{2} \nabla(\boldsymbol{u} \cdot \boldsymbol{u})-\boldsymbol{u} \wedge(\nabla \wedge \boldsymbol{u})
$$

are advanced according to the splitting scheme:

$$
\frac{\tilde{\boldsymbol{u}}-\boldsymbol{u}^{n}}{\Delta t}=\sum_{q=0}^{K_{e}-1} \beta_{q} \boldsymbol{N}\left(\boldsymbol{u}^{n-q}\right)
$$

$$
\frac{\boldsymbol{u}^{*}-\tilde{\boldsymbol{u}}}{\Delta t}=-\nabla \bar{p}^{n+1}
$$




$$
\frac{\boldsymbol{u}^{n+1}-\boldsymbol{u}^{*}}{\Delta t}=v \sum_{q=0}^{K_{i}-1} \gamma_{q} \boldsymbol{L}\left(\boldsymbol{u}^{n+1-q}\right)
$$

Here $K_{e}$ and $K_{i}$ refer to the order of an explicit and implicit time integration scheme with associated coefficients $\beta_{q}, \gamma_{q}$. The first step integrates the nonlinear advection terms using a multi-level Adams Bashforth scheme. In the second step the timeaveraged pressure is recovered by solving the Poisson equation obtained from the second relation above, with the assumption that $\nabla \cdot \boldsymbol{u}^{*}=0$, and with boundary conditions which ensure the splitting error is consistent with the overall temporal discretisation. Finally the last step is rearranged into a Helmholtz equation for each velocity component, with an implicit treatment for the viscous terms, using either backwards Euler or Crank-Nicolson schemes denoted by the coefficients $\gamma_{q}$.

The mesh generation used a modified advancing layers technique for the near wall region (Peiró and Sayma, 1995), and a method based on the advancing front technique for the rest of the domain, (Peraire et al 1993). The resulting mesh of linear tetrahedral elements was then transformed into a boundary conforming mesh of highorder elements. An eight order polynomial basis was used for both model graft cases, corresponding to local degrees of freedom of 287,000 and 320,000 for the planar and non-planar grafts respectively, with 1742 elements for the planar model and 1941 elements for the non-planar model. For the coronary graft model, a combination of more elements (2469) and a lower polynomial order of 6 provided 291,000 local degrees of freedom.

\subsection{Computational methods for vortex identification}

Isosurfaces of negative values for the second largest eigenvalue of the matrix $\left[S^{2}+\right.$ $\boldsymbol{\Omega}^{2}$ ] were used to identify vortex cores embedded in the flow, whereas particle tracking was used to qualitatively model the dynamics of vortex generation and evolution. The coefficients of the velocity gradient matrix at each interpolant node were determined, and a standard QR routine in LAPACK was used to determine the eigenvalues. From this the isosurface of the second eigenvalue could be rendered. 
Tracking of marker particles for computations with the spectral $h / p$ finite element formulation used a routine which took advantage of the high order spatial discretisation. Integration solely in physical space is computationally expensive for high order elements; if $\mathcal{X}^{\mathrm{e}}$ denotes the mapping from physical space coordinates $\boldsymbol{x}$ to those in parametric element coordinates $\zeta$, i.e. $\mathcal{X}^{\mathrm{e}}(\zeta)=\boldsymbol{x}$, finding the element coordinates which correspond to a given physical location, $\boldsymbol{x}_{o}$, is a non-linear problem requiring iterative solution. Let the initial estimate of the particle parametric coordinates be $\zeta_{i}$ and let $\boldsymbol{F}\left(\boldsymbol{\zeta}_{i}\right)=\mathcal{X}^{\mathrm{e}}\left(\boldsymbol{\zeta}_{i}\right)-\boldsymbol{x}_{o}$, then the Newton-Raphson sequence:

$$
\zeta_{i+1}=\zeta_{i}-\frac{\boldsymbol{F}\left(\boldsymbol{\zeta}_{i}\right)}{(\partial \boldsymbol{F} / \partial \zeta)}=\zeta_{i}-\frac{\boldsymbol{F}\left(\boldsymbol{\zeta}_{i}\right)}{J_{e}}
$$

should rapidly converge provided the time step is appropriately small for accuracy, where $\partial \boldsymbol{F} / \partial \boldsymbol{\zeta}=\partial \mathcal{X}^{\mathrm{e}} / \partial \boldsymbol{\zeta}=J_{e}$. The iteration however requires interpolating the element mapping Jacobian $J_{e}$, which is a significant computational cost.

Tracking the particles entirely within the parametric representation for each cell appears to be an attractive alternative, especially since the cell dimensions are relatively large for the high order $h / p$ formulation. To be fully consistent, this requires a solution of the non-linear problem of determining the time at which the particle crosses from one element face to a neighbour and employing a variable step integration routine with appropriately adjusted time step. The Jacobian of the mapping and hence the velocity field in parametric coordinates may however vary rapidly near the element boundary and be ill-behaved outside it, in zones where the elements are distorted. There is also a singular point associated with the mapping of the orthogonal coordinate system to a tetrahedron which may produce further numerical difficulties. Thus determining the element crossing time is not straightforward.

In Coppola et al (2001) alternative schemes for tracking in high-order elements are developed and compared. The most efficient described is a hybrid scheme; this primarily employs explicit Runge-Kutta integration to advance the particle trajectories 
in parametric coordinates within each high order cell coupled to a guided search algorithm to allow particles to be tracked from cell-to-cell without transforming to and from physical coordinates. The transition from cell to cell can introduce errors, since the Jacobian of the mapping is non-constant in high-order elements. It is however simple to map from parametric coordinates to physical co-ordinates, so the sub-steps of the integration can be evaluated in parallel in both physical and parametric coordinates and compared as an error check. Adaptive correction of the particle trajectory can then be obtained by reverting to full physical coordinates for steps where the error is outside the required tolerance.

For comparison with the $h / p$ results, steady flow computations were also performed using the commercial package FLUENT with second order upwind discretisation on a hexahedral mesh. It was possible to achieve good agreement with the h/p results, though the FLUENT computations used over 1 million elements (1.1 million for the planar and 1.4 million for the non-planar grafts respectively). Using this velocity field representation, each hexahedral element is transiently decomposed into tetrahedral sub-elements to evaluate the particle velocity. This avoids producing a very large static element decomposition file, and it is also more straightforward to assure that as a particle transits from one element to the next, the decomposition of adjacent elements maintains consistent diagonal face splittings. Whereas the exact trajectories for steady flow, $\boldsymbol{x}=\boldsymbol{x}_{\boldsymbol{0}} \exp [(\nabla \boldsymbol{u}) t]$ could be employed within each such element (considered in Doorly \& Franke (2001)), a fourth order Runge-Kutta scheme was again used.

\subsection{Flow structure in end-to-side grafts}

In the host artery flow downstream of the anastomosis, the dominant feature which emerges is a pair of counter-rotating vortices. The vortex dynamics model of section 2.3 for this configuration is sketched in fig. 2. Each ring representing the mean vorticity in an element of the graft impacts the host artery and is distorted by stretching as it exits the host. This model of flow is analogous to the problem of an oblique vortex impact on a plane wall (Orlandi 1993, Doorly \& Liu 1995), though the confining presence of the side walls in this case greatly enhances the vortex stretching of the two lateral 'arms' of the vortex in the exit flow direction. Isosurfaces for a 
value of the eigenvalue $\lambda_{2}$ (section 2.2) slightly below zero (henceforth simply referred to as the $\lambda_{2}$ isosurface) are shown in figure 3, and the main isosurface clearly demonstrates the twin vortex cores. Detailed examination of the figure suggests a connection between the cores at the junction; also revealed are other small isosurfaces, located on the bed of the artery, and at the inner corner towards the occluded end.

Figure 4 shows the position of a material line with radius $3 / 4$ of the pipe both initially in the graft, and at some time after it has entered the host artery. Comparing the different approaches, the $\lambda_{2}$ isosurface should locate the vortex structures accurately since it is obtained from a local approximation to the velocity field. (Note however that the minimum value of $\lambda_{2}$ will not generally correspond to the centre of rotation in a plane, rather it identifies the point where the relative rotational acceleration is strongest. This is because the most negative contribution comes from $\boldsymbol{\Omega}^{2}$ away from a boundary and is equal to $-1 / 4|\omega|^{2}$ ). The $\lambda_{2}$ isosurface only indirectly takes account of vorticity generation at a boundary, and does not indicate how the boundary vorticity can be connected to interior structures. This is because near the wall, the flow is forced to become aligned with the wall as is the vorticity, and rotation is suppressed. A more serious limitation of identification based solely on the $\lambda_{2}$ isosurface is that it provides little indication of the dynamics of the processes which lead to the creation of the vortex flow structures in the flow interior.

On the other hand, tracking the evolution of material rings and appealing locally to the Helmholtz theorem is admittedly rather qualitative. Factors such as the coarseness of the lumped representation, and the problem of non-convective transport of vorticity by viscous diffusion provide a degree of uncertainty in the correspondence between material lines and vortex cores. Furthermore, unless the inlet flow vorticity can be represented by a simple structure, for example a ring or series of rings, relating material line distortions to the vorticity evolution is extremely difficult. Where the inlet flow is simple, as in this case, tracking appropriately placed material lines or rings clearly identifies regions of rapid stretching. (Projecting the local values of $\boldsymbol{\omega} \cdot \nabla \boldsymbol{u}$ onto the marker points, which comprise the material filament, could provide more quantitative information). The tilting and stretching mechanisms, which 
generate the vortex pair in the host vessel in this flow, are very well illustrated, as is also the stagnation of the flow on the 'bed' of the host artery.

Using an additional material ring at $1 / 4$ the pipe radius indicates how the core flow behaves, fig. 5. As can be seen, the core flow, which has little vorticity, simply impacts on the artery bed. This brings it into the near wall region, where it is gradually distorted in a fresh boundary layer generated in the host. Particularly striking in the figure is the lack of mixing between simultaneously released core and outer rings.

\subsection{Vortical flow structure in non-planar graft geometry.}

The geometry of the graft strongly affects the flow in the anastomosis region, as expected from studies of the sensitivity of arterial flow to 3D geometry (Caro et al 1996). In grafts many studies have looked at effects of parameters including 3-D curvature, graft to host diameter ratio, and graft angle on the flow. In this part we apply the vortex identification and tracking techniques described above to investigate how 3-D curvature affects the flow; the procedure could also be used to examine the flow consequences of other parameters.

A comparable series of images to those for a symmetric planar graft are shown in fig. 6 for a graft where the inflow is rotated 90 degrees out of the plane of the junction, (Sherwin et al 2000). As the outer ring traverses along the curved graft it is initially tilted forwards on the inner wall where the flow follows a shorter path. This results in rapid stretching which produces streamwise components of vorticity (the Dean vortices) and alters the velocity field. The altered velocity field has little upstream extent so that representing the inflow as a series of rings remains justified. Downstream in the bend, the initial ring is further distorted by the developing velocity field. As the lateral portions of the material ring do not correspond exactly to the vortex cores, they are slightly twisted. From the degree of stretching it is apparent that the Dean vortices should be of appreciable strength, and eventually vortex augmentation by stretching reaches a balance with the viscous diffusion.

The outer ring enters the graft asymmetrically, and the side which first approaches the wall of the host vessel is more rapidly stretched and eventually dominates the 
downstream flow. The inner ring is tilted and displaced towards the outer wall in the bend region by the vortical flow. There it is stretched in the streamwise direction whilst the crossflow velocities induced by the Dean vortices cause opposite the sides to begin to wrap around the circumference. In this case both filaments are very highly stretched in the graft, and become enfolded. Stretching and folding are primarily responsible for mixing in flows with negligible diffusion. Periodic reorientation of the flow is known to dramatically increase mixing efficiency (defined in Ottino 1989). Fig. 6 also illustrates how the reorientation of the vortex structures from the graft to the host exit flow leads to the enfolding and stretching of both initial rings.

Comparison of the magnitude of the velocity components for the flow entering the host for this non-planar geometry shows that the peak axial velocity is nearly an order of magnitude larger than the transverse components. In that case one may suggest that the primary effect of the graft curvature is to generate an asymmetrical streamwise velocity profile. The asymmetrical profile enters the host vessel below the centreplane and should therefore set up a swirl velocity in analogous fashion to a cyclone separator. To investigate this we have computed the flow for a planar configuration, but with a non-uniform inlet velocity profile. This "modified inflow" has axial velocity chosen to match that produced at the exit of the bent portion of the graft, and the cross-flow components are set to zero. (The modified inflow corresponds to that which could be produced by placing a filter with a resistance varying in $(r, \theta)$ in the inflow close to the anastomosis). Fig. 7 compares the streamwise component of the flow which develops at three diameters downstream of the anastomosis for the nonplanar graft, and for the planar graft with the modified inflow. The agreement is remarkably good, indicating the major effect of the non-planar inflow is indeed due to the displacement of the axial velocity profile. Fig. 8 which compares the vortex structures for the non-planar geometry and the modified inflow also shows close agreement.

The primary effect of the Dean vortices which develop in the graft appears therefore to be that they distort the azimuthal vorticity distribution, and that the injection of this distorted vorticity leads to the observed primary swirl direction downstream. Tracking appropriate material lines supports this but for brevity we omit the details. 
Interestingly however, the dominance of one vortex over another is greater in the nonplanar case than for the modified inflow, as can be observed in fig. 8. This may be related to the connection between the Dean vortices at the exit of the graft, and the vortex structures downstream of the junction as shown in fig. 9. Consider first the Dean vortex of the pair which is rotating clockwise along the flow direction. It undergoes a further clockwise rotation as it develops in the graft and strengthens. Conversely the vortex which is rotating anticlockwise in the graft is diminished as it is forced to turn clockwise to assume its position downstream in the host vessel. The modified inflow does not posses any streamwise vortices initially, so it does not share this mechanism. This may explain the lesser dominance of one vortex in the modified inflow vis a vis the non-planar graft.

Removing the portions of the $\lambda_{2}$ isosurface outside a section between one and two diameters downstream of the toe indicates the positions of the vortex cores for the three cases: (i) planar graft, (ii) planar graft with modified inflow and (iii) non-planar graft, as shown in fig. 10. The modified flow is again seen to represent an intermediate stage between the planar and non-planar configurations. Moving from (i) to (iii) in the figure it is evident that as the clockwise rotating vortex becomes more dominant it moves closer to the axis, as expected.

\subsection{Vortical structures in unsteady flow.}

The variation in the vortical structures and wall shear stress in unsteady flow through the planar model graft is shown in fig. 11 for a Womersley number $\alpha=a \sqrt{ }(2 \pi f / v)$ of 4. At the graft inflow a Womersley velocity profile was prescribed comprising a single harmonic superimposed on a steady flow with mean Reynolds number 250. The bulk flow velocity does not reverse and has a minimum of $1 / 4$ that of the peak. Examining fig. 11 clockwise from the upper left corner, plot (i) corresponds to the flow minimum when the vortex structures are weakest. Plot (ii) shows the structures extending and the corresponding shear map during peak acceleration. During the subsequent quarter cycle the flow resembles the steady case, since the acceleration reduces (and is relatively weak given that the ratio of flow period to convective timescale $(u / d f)$ is $\sim 24)$ and the velocities are approximately comparable. At peak velocity (iii) the vortex structures extend their maximum distance upstream into the 
graft portion, which is still only of the order of a diameter, and the deceleration begins. Finally plot (iv) in decelerating flow reveals a curved or wave-like displacement of the vortex cores, which appears at the same time as the development of reversed flow regions on the upper portion of the host vessel near the toe region.

The trajectories of a ring (with diameter $3 / 4$ that of the graft) of particles released at different times in the graft are shown in fig. 12. (Strictly the initial ring diameter should vary to reflect the mean location of vorticity in the particular phase of the Womersley cycle). Image (A) corresponds to release just before the flow minimum; this ring enters the graft just after the start of the cycle as the acceleration develops. For image (B), the release time is such that the ring enters the graft at about the midpoint in the increasing velocity phase, where the acceleration just diminishes to zero. The flow conditions at this point are closest to steady flow and the ring at 7T/16 appears similar to that shown in figures $4 \& 5$. For images (C) and (D) the flow is decelerating, and the particle positions reflect the undulation of the vortex cores observed in the structure plot of fig. 11 (iv).

\subsection{Application to real graft geometry}

The particle tracking and structure identification have been applied to a coronary bypass graft in steady flow, figure 13 . The graft was surgically constructed ex-vivo using a porcine heart and human saphenous vein. The entire coronary geometry was cast at a pressure of $80 \mathrm{~mm} \mathrm{Hg}$, the surrounding tissues were dissolved and the cast scanned using MRI with a slice thickness of 1mm (Papaharilaou et al, 2001). From this a computational mesh was constructed, which was then also used to produce a stereolithographic model - comparison of this and the original cast verified the high degree of fidelity of the computational mesh. For the computational model, a straight outlet portion was blended to the host artery at three diameters downstream of the toe. The anastomosis geometry takes the form of a stretched $\mathrm{S}$ bend, which appears almost planar. There is however a noticeable pinching of the graft where it joins the artery which accelerates the flow. The acceleration appears to magnify the effect of the slight out-of-plane curvature since the flow downstream in the host is asymmetric and dominated by a single vortex core. 
Fig. 13 shows the position of a material ring in a sequence of equi-spaced intervals labelled 1-5 in 3 orthogonal corresponding views. In the upper left portion of the plot, the vortical structure from the $-\lambda_{2}$ isosurface is included. In the head on view, top, the portions of the isosurface outside a section between $1 \frac{1}{2}$ and 3 diameters downstream of the toe have been removed revealing the core centre which is filled with a dark colour and marked with a central dot. Examining the images for the ring, those labelled 1 correspond to a time shortly after release of the ring, and whereas the set of images 2 show the ring being tilted and stretched as the flow curves through the bend in the graft. At this point, the lower portion of the ring is markedly in advance, and is already undergoing a pinching and acceleration through the narrowing at the anastomosis. The third set of images demonstrates that a rapid transformation has occurred; the portion of the ring on the right (arrowed in the figure) has undergone a rapid stretching and has moved across the bed region of the host to the left side. Stretching of this portion of an initial vortex ring establishes the dominant anticlockwise rotation of the flow (in the streamwise direction) which is evident just downstream of the anastomosis. Frames 4 and 5 show the continued distortion and stretching of the ring in the outflow; note that in the upper sequence showing the graft head-on looking upstream, the anticlockwise rotation appears clockwise,

\section{Haemodynamic particle transport by the flow.}

The preceding methods for investigating the vortex dynamics of the flow lead naturally to studying the transport of molecular species and particles such as red blood cells and platelets. Vortical structures play a key role in the transport process both by controlling the mixing of particles across the vessel section, and in the control they exert on the dynamics of reversed flow regions in which particles may experience long residence times.

\subsection{Effect of vortical structures on particle residence time in graft.}

A crude overall measure of the effective residence time for particles in the region of the graft may be obtained by computing the exit time distribution for a set of particles to pass from the inflow to a section downstream of the graft junction. Results for steady flow at $\operatorname{Re} 250$ are shown in figure 14, which compares the temporal distributions for approximately 70,000 particles passing a section 3 diameters 
downstream of the graft 'toe' for the planar and non-planar idealised models. The particles were initially uniformly distributed across the section as a disk comprising nested rings, and the disk was released just upstream of the entrance to the bend in the non-planar geometry, and at 2.5 diameters upstream of the 'toe' in the planar geometry. Each distribution is compared with that expected for Poiseuille flow, over a length equal to that of the geometric centreline path from the graft release location to the measuring plane at 3D. Time is normalised by the median arrival time for particles in Poiseuille flow over the equivalent length. For Poiseuille flow, it is straightforward to show that the arrival rate of particles $d n / d t^{\prime}$ is given by

$$
\frac{d n}{d t^{\prime}}=\frac{1}{\left(t^{\prime}+1\right)^{2}}
$$

where $t^{\prime}$ is measured from the arrival of the particle with highest (centreline) velocity.

For the planar geometry, the arrival time for the graft particles almost exactly matches that for the Poiseuille flow, and the overall shape of the distributions is similar. The results suggest that the gross mixing characteristics of planar graft flow are not greatly altered from those of Poiseuille flow, though differences are seen in the 'tail' of the distributions. In the planar graft, the vortical flow structures have only become established over a relatively short distance before the measurement plane at 3D, and these structures have not displaced the large bulk of particles to regions with much different axial velocities. By contrast, for the non-planar case, the vortical structure developed in the bend flow is reorientated as the flow develops in the graft, with consequently significantly greater mixing of particle trajectories

\subsection{Modelling reactions between particles and the flowfield.}

Apart from studying the transport and trajectory dynamics of particles however, we would also like to model the effect of flow-induced forces (Aarts et al 1986) and biochemical environments on them. These are important determinants for the potential interaction of particles such as platelets with the wall and with other particles. 
It is well recognised that a number of chemical agonists can induce or inhibit platelet activation, and that activation may also be affected by fluid shear, e.g. effects of ADP as reported by Goldsmith et al (1994). The effects of exposure to fluid shear stress on platelets and red blood cells depends on both the stress level and the duration of exposure (Hellums 1987, Boreda et al. 1995). The mechanisms responsible are unclear; fluid mechanical forces are known to influence cell morphology and if sufficiently large can destroy cells. Increased shear stress likewise provokes a greater degree of mixing between fluid layers and more effective transport of potential agonists to the cells. It is perhaps most likely that both phenomena act in concert. Whether considering either or both mechanisms, Lagrangian tracking of fluid particles and their interaction with their environment is desirable.

To quantify the fluid shear stress accumulation, for the present we have used the Von Mises criterion as described by Bludzuweit (1994), which is related to failure due to shear, i.e. we compute the linear rate of accumulation of $\sigma_{e}$ along the trajectory, where

$$
\sigma_{e}=\frac{1}{\sqrt{2}}\left\{\left(\sigma_{x x}-\sigma_{y y}\right)^{2}+\left(\sigma_{y y}-\sigma_{z z}\right)^{2}+\left(\sigma_{x x}-\sigma_{z z}\right)^{2}+6\left(\sigma_{x y}^{2}+\sigma_{y z}^{2}+\sigma_{x z}^{2}\right)\right\}^{1 / 2}
$$

Boreda et al (1995) proposed the calculation of a platelet stimulation function, PSF, defined as:

$$
P S F=\tau t^{0.452}
$$

where $\tau$ represents the magnitude of the shear stress, $\left(\sigma_{e}\right.$ could arguably be used instead) and $t$ the exposure time. For $\tau$ measured in dynes $/ \mathrm{cm}^{2}$ and $t$ in seconds, they give a value of 1000 as the shear activation threshold from the data of Hellums et al (1987). However their measurements of flow through a stenosed artery model indicated PSF values one to two orders of magnitude below this. Although this suggests that in most physiological situations, shear alone may not be sufficient it is considered to be a very significant factor, (Wootton \& Ku (1999)). We confine ourselves here to study the relation between the flow dynamics and fluid shear; biochemical interactions are considered elsewhere (Franke and Doorly, 2001). 
The vortical structures are primarily responsible for mixing of species with low diffusivity. Interaction with the red blood cells, which are induced to translate and rotate by velocity shear, may increase the diffusivity of platelets many orders of magnitude above the Stokes-Einstein thermal prediction, $\mathrm{O}\left(10^{-9} \mathrm{~cm}^{2} \mathrm{~s}^{-1}\right)$, to effective diffusivities of $\mathrm{O}\left(10^{-7} \mathrm{~cm}^{2} \mathrm{~s}^{-1}\right)$ at shear rates of $100 \mathrm{~s}^{-1}$ and as high as $\mathrm{O}\left(10^{-5} \mathrm{~cm}^{2} \mathrm{~s}^{-1}\right)$ at shear rates of $10,000 \mathrm{~s}^{-1}$. Particle segregation effects suggest furthermore that the effective diffusivity may not be isotropic and some models attempt to incorporate this, Eckstein and Belgachem (1991). In any event the Péclet number which relates convective and diffusive transport is always very large, and over local regions of several diameters, mixing in large arteries is dominated by the flow kinematics. The interrelation between the flow dynamics, mixing and the normalised shear exposure of particles is illustrated in figures 15 and 16. (The accumulated linear shear is divided by that experienced by the median particle in the equivalent Poiseuille flow). In figure 15 , the effect of the vortical flow structure on mixing via dispersion is compared for the two geometries at different Reynolds numbers by plotting the shear exposure of particles crossing a section at 3D downstream of the toe. Results from each geometry show that the vortical structures play a more vigorous role in mixing as the Reynolds number increases. Also clear is the more complete mixing of the particles for the nonplanar geometry.

The highest particle density corresponds to the high velocity region, which for both cases corresponds to a displaced crescent. The particles which have experienced the steep velocity gradients near the wall have highest accumulated shear exposure. There is a concentration of high shear particles near the part of the wall where the boundary layers erupt; at higher $R e$ the eruption is more developed, and the high shear particles are rapidly swept into the mainstream. For this geometry the vortical structures in the bend, and the re-orientation and alteration of the vorticity in the host have produced appreciable mixing of the high shear particles into the core flow. The transport of particles away from the wall helps to lessen the residence time, which can be used as a comparative indicator of the mixing efficiencies of different geometries. Comparison of both the mean residence time, and that of highly shear loaded particles with diffusion may be compared to predictions from Taylor dispersion for straight or curved tubes Pedley \& Kamm (1988), but we postpone discussion of this to further work. 


\subsection{Effects of diffusion on Lagrangian Dynamics}

Given the low value of diffusion for large molecular species and small particles such as platelets, one may expect the particle dynamics to closely follow that obtaining with zero diffusion. Up to a point this is correct, as shown in parts (a) and (b) of figure 16 , where a detail of the shear exposure distribution at 3D for the planar graft flow at Re 250 is compared without and with diffusion. However in certain regions of the flow, the Lagrangian particle trajectories are very sensitive to initial conditions; though diffusion may be very small, the envelope of possible trajectories of a particle passing through a point can then spread very widely. Even for steady flow therefore one cannot ascribe a single point of origin (with a corresponding stress exposure along the trajectory) with certainty to a particle arriving at a specific location near the wall. Instead the deterministic trajectory is replaced by a probability distribution over a bundle of trajectories. In Poiseuille flow the bundle would correspond to a cone (with apex angle proportional to the Schmidt number) of trajectories about a central, non-diffusive limit. With three dimensional and unsteady flows the probable bundle of trajectories may become highly convoluted, may even break apart if the dynamics becomes chaotic. As an illustration figure 16 (c) shows three possible trajectories of particles released from a point 2 diameters upstream of the anastomosis for the nonplanar graft of section (3.2). The trajectories each enter the recirculation region for a time and exit with considerable variation in their shear exposure. Figure 16 (d) illustrates the considerable spread in exit points which is possible even for such small values of diffusion.

Considering the local haemodynamics in large arteries, the majority of particles are transported through without the possibility of interaction with the wall. In modelling particle-wall interaction therefore, a computationally efficient procedure is to use the flow solution to identify regions of interest, for example stagnation points, recirculation zones etc. which are seeded with particles. The probable condition of these particles can then be computed by tracing the corresponding Wiener bundle of trajectories backwards (Doorly 1999 ), to assess the likely state of the particles which arrive near the wall as a consequence of their previous history of interactions with the flow. This provides an initial condition for the particles; the particle-wall interaction 
can then be simulated using appropriate models (David et al 1999) for the nearsurface flow and the biochemical environment.

\section{Conclusions}

The work reported here concentrates on the large scale dynamics of the flow and its impact on particle transport. It has been demonstrated that vortex structure identification and vortex modelling reveals much of the important flow dynamics and its consequences for transport and mixing. A probabilistic treatment of particle wall interaction seems best suited to describe the state of particles given the effects of diffusion and the possibility of widely diverging trajectories. This paper describes a basic outline for computational modelling but there is much scope for further improvement including: a proper treatment of the near wall interaction, inclusion of rate reactions for particles, and more experimental data on the mechanisms of platelet activation.

\section{Acknowledgements}

We are grateful to The BUPA Foundation and The Clothworkers' Foundation for their support of this work.

\section{References:}

Aarts, P.A.M.M., Steendijk, P., Sixma, I.J., and Heethaar, R.M. (1986). Fluid shear as a possible mechanism for platelet diffusivity in flowing blood. J. Biomechanics, 19, pp. 799-805.

Agrawal Y, Talbot L and Gong K (1978). Laser anemometry study of flow development in curved circular pipes. J. Fluid Mech., 85 497-518.

Boreda R, Fatemi RS, Rittgers, SE (1995). Potential for platelet stimulation in critically stenosed carotid and coronary arteries. J. Vasc Invest, 1(1):26-37 
Bludszuweit C., (1994). PhD thesis, Univ. of Strathclyde, Glasgow.

Buchanan, J.R., and Kleinstreuer, C. (1998). Simulation of particle hemodynamics in a partially occluded artery segment with implications to the initiation of microemboli and secondary stenosis. J. Biomech. Eng., 120, pp. 446--454.

Caro C. G, Doorly D. J., Tarnawski M., Scott K. T., Long Q. and Dumoulin C.L. (1996). Non-planar curvature and branching of arteries and non-planar-type flow, Proc. Roy. Soc. (A), 452, 185-197

Coppola G, Sherwin S, and Peiró J. (2001) Nonlinear particle tracking for high order elements. J. Comp. Physics 172, 1-31

David T, Thomas S, and Walker P.G. (1999) Platelet deposition in stagnation point flow: an analytic and computational simulation, pp49-58 in Cardiovascular flow Modelling and Measurement, Ed. S. G. Sajjadi, G. B. Nash, M. W. Rampling. Oxford ISBN 0198505205

Doorly D.J., and Liu, C.H. (1995). Tracking the development of vortex flow structures using vortex particle-in-cell methods, in Numerical Methods for Fluid Dynamics V, Editors: K.W. Morton and M.J. Baines, Oxford.

Doorly D J (1999) Modelling of flow transport in arteries pp 67 - 81 in Cardiovascular flow Modelling and Measurement, Ed. S. G. Sajjadi, G. B. Nash, M. W. Rampling Oxford ISBN 0198505205

Doorly DJ and Franke PT. (2001) Computational modelling of particle transport in blood flow, in preparation

Ethier CR, Prakash S, Steinman DA, Leask RL, Couch GG and Ojha M. (2000). Steady flow separation in a 45 degree junction, J. Fluid Mech. 411 1-38

Eckstein, E.C., and Belgacem, F. (1991). Model of platelet transport in flowing blood with drift and diffusion term. Biophysical J., 60, pp. 53-69.

Franke PT and Doorly DJ. (2001). Unsteady flow effects on transport in artificial vascular devices, in preparation.

Goldsmith H L, Frojmovic MM, Braovac S, McIntosh F, and Wong T (1994). Adenosine diphosphate-induced aggregation of human platelets in flow through tubes III. Shear and extrinsic fibrinogen dependent effects. Thromb. Haemost. 71 (1):78-90

Hellums JD, Peterson DM, Statopoulos NA, Moake JL, Gorgio TD. (1987). Studies on the mechanisms of shear-induced changes in platelet activation, in Hartmann A. Kuschinsky W, eds. Cerebral Ischemia and hemorheology. Springer-Verlag Berlin 8089.

Hofer M, Rappitsch G, Perktold K, Trubel W, Schima H, (1996). Numerical study of wall mechanics and fluid dynamics in end-to-side anastomoses and correlation to intimal hyperplasia. J. Biomech 29, pp 1297-1308. 
Jeong \& Hussain (1995) On the identification of a vortex, J. Fluid Mech. 285, 69-94

Orlandi, P and Verzicco R., (1993). Vortex rings Impinging on walls axisymmetric and 3D simulations. J. Fluid Mech. 256 pp 615-645.

Ottino JM. The kinematics of mixing. (1989) Cambridge University Press.

Papaharilaou Y, Doorly D. J., \& Sherwin SJ (2001) Assessing the accuracy of twodimensional phase-contrast MRI measurements of complex unsteady flows, to appear in J. MRI

Pedley, T.J. \& Kamm, R.D. (1988) The effect of secondary motion on axial transport in oscillatory tube flow, J. Fluid Mech. 193 347-367

J. Peiró and A. I. Sayma, (1995). A 3-D Unstructured Multigrid Navier-Stokes Solver, in Numerical Methods for Fluid Dynamics V, (K. W. Morton and M. J. Baines, Eds.), Oxford University Press, (1995)

Peraire J, Morgan K and Peiró J. (1993)Multigrid Solution of the 3D Compressible Euler Equations on Unstructured Tetrahedral Grids, Int. J. Num. Meth. Engng., 36, 1029-1044,.

Saffman PG, (1995). Vortex Dynamics. Cambridge University Press. ISBN 0521477395

S.J. Sherwin S.J. and Karniadakis G.E., (1996) Tetrahedral hp Finite Elements: Algorithms and Flow Simulations, J. Computational Physics, 124 14-45

Sherwin S. J. and Karniadakis G.E. (1999) Unstructured hp Finite Element Methods for Computational Fluid Dynamics, Oxford University Press, ISBN 0-19-510226-6

Sherwin S.J, Shah O, Doorly D. J., Peiro J., Papaharilaou Y, Watkins N, Caro C G and Dumoulin C.L.(2000). The influence of out-of-plane geometry on the flow within a distal end-to-side anastomosis , ASME Journal of Biomechanical Engineering.122 1-10

Steinman, D. Frayne R, Zhang XD, Butt B, Ethier C (1996) MR measurements and numerical simulation of steady flow in an end-to-side anastomosis model. J. Biomech 29, no. 4 pp 537-542.

Wootton, D.M and Ku, D. N. (1999). Fluid Mechanics of vascular systems, diseases and thrombosis. Ann. Rev. Biomed Eng. 01:299-329.

White S, Zarins C, Giddens D, Bassiouny H, Loth F, Jones S, Glagov S, (1993), Haemodynamics patterns in 2 models of end-to-side vascular graft anastomoses: effect of pulsatility, flow division, Reynolds number and hood length. ASME J. Biomech Eng,

114 , pp104-115. 


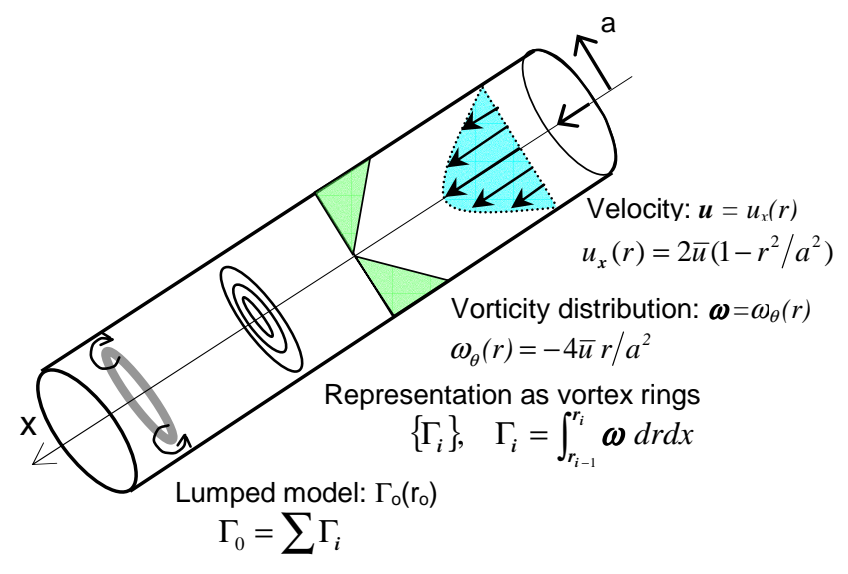

\section{Figure 1}

Vorticity distribution and vortex model for pipe flow.

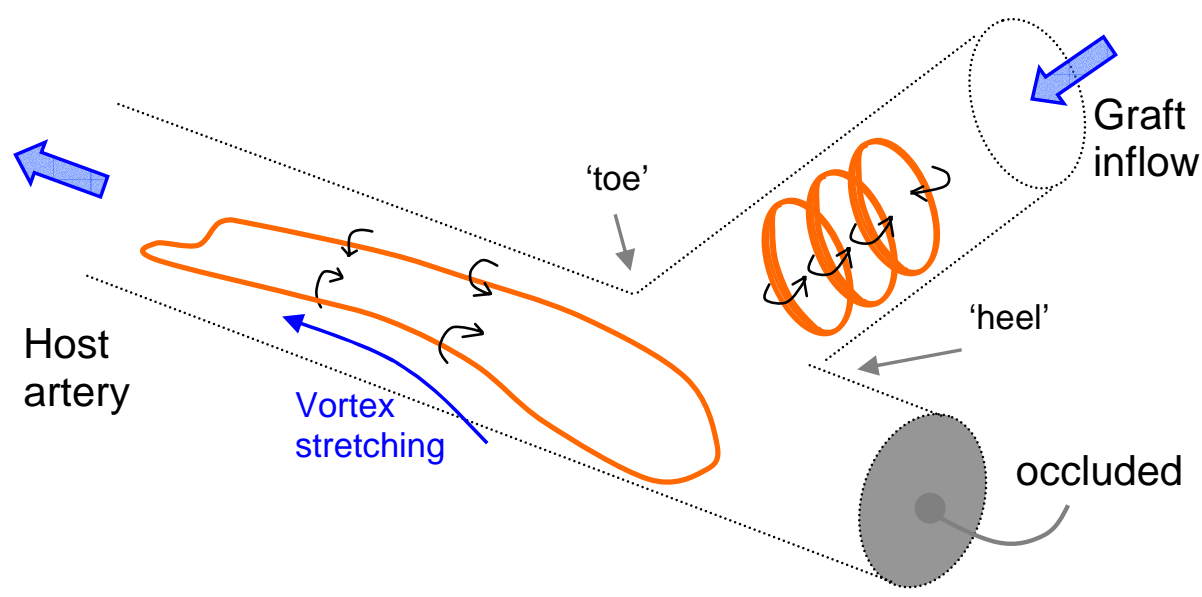

\section{Figure 2}

Flow in idealised end-to-side anastomosis model. The vortex dynamics model represents the vorticity in the graft as a series of rings which become highly stretched after they enter the host vessel. Vortex stretching leads to intensification of vorticity and produces a pair of counter-rotating vortices in the distal flow. 


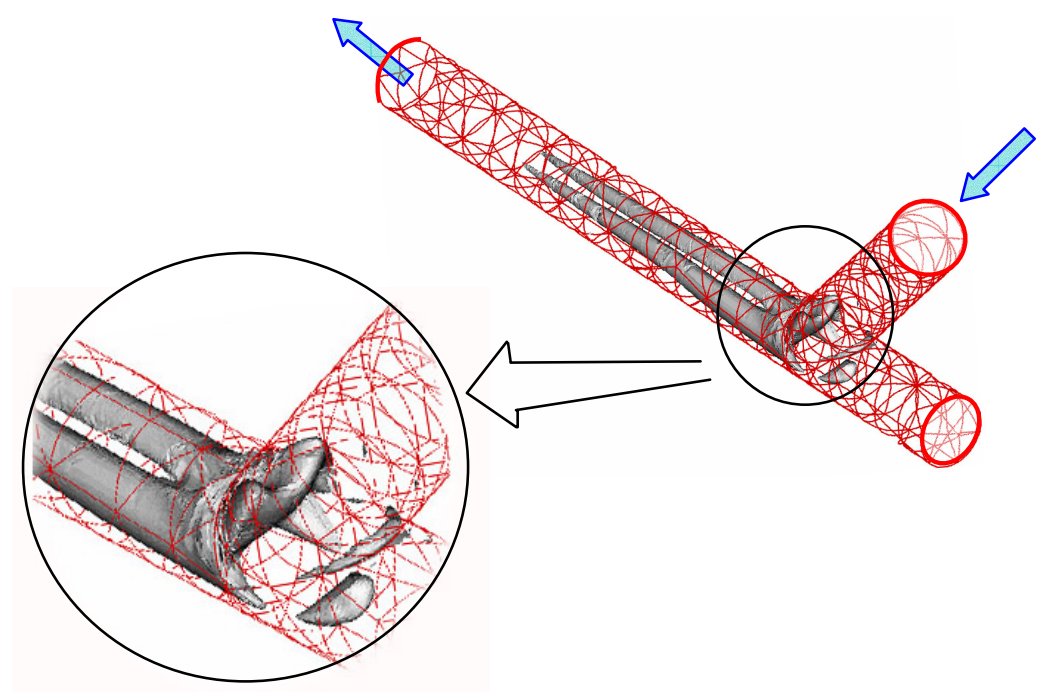

Figure 3.

Flow structures in bypass graft in steady flow at Re 250 revealed by isosurface of $\lambda_{2}<0 ; \lambda_{2}$ is second largest eigenvalue of $S^{2}+\Omega^{2}$ (symmetric part of $\left.(\nabla \mathrm{u})^{2}\right)$
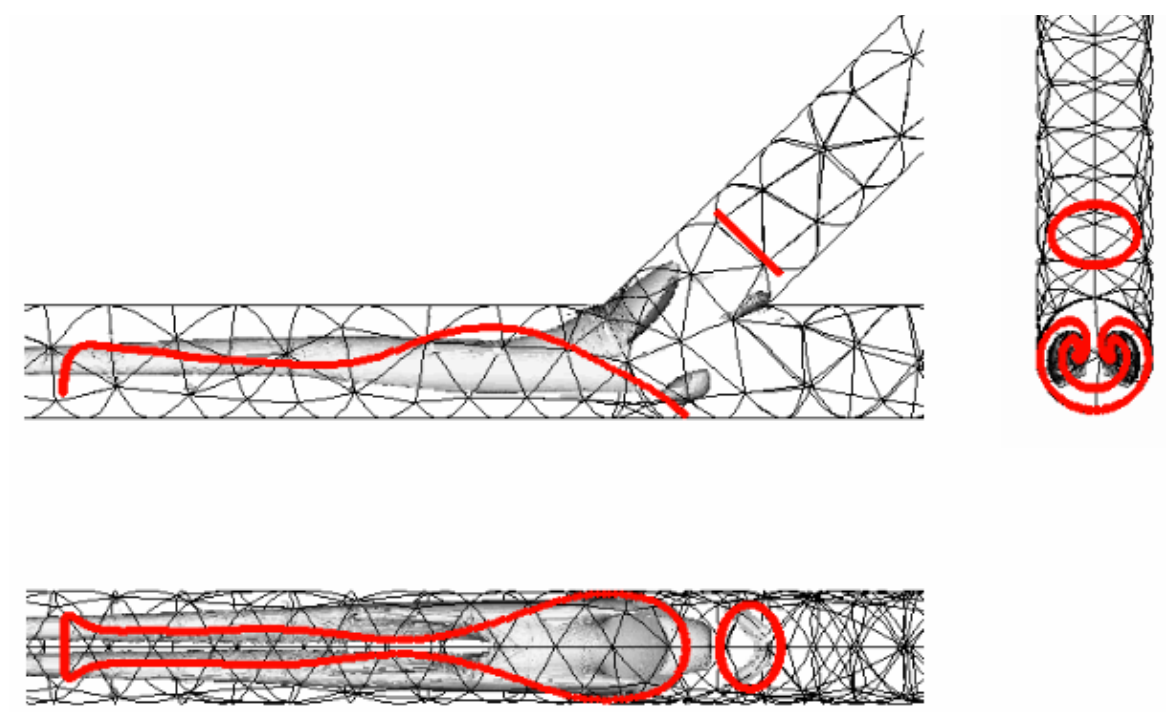

\section{Figure 4}

Comparison between vortical flow structures in bypass graft and visualisation using marker particles. Ring of particles released in graft shown in two positions: a) just after release, b) after entering host. 

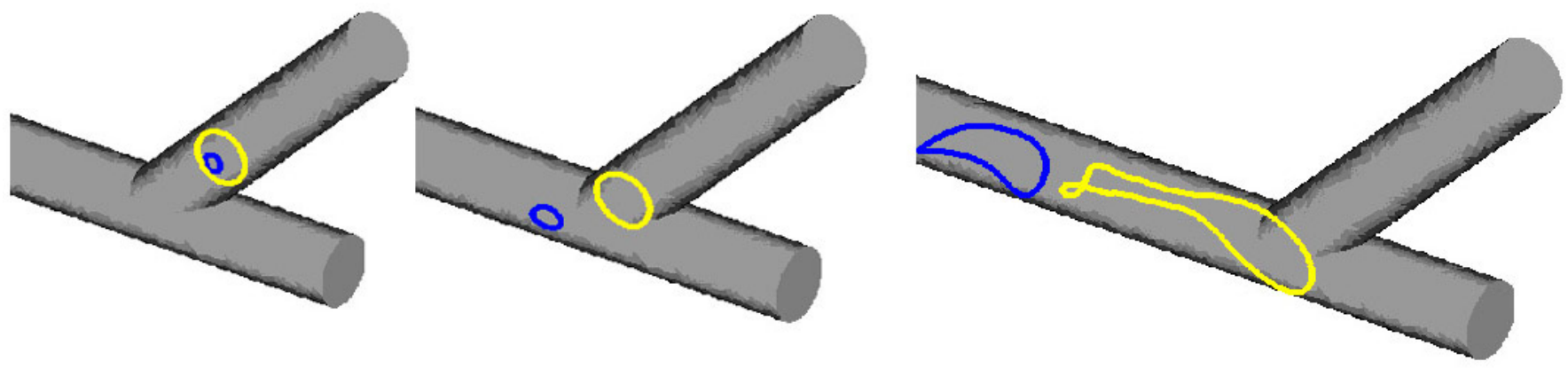

Figure 5

Transport of marker particle rings in planar model end-to-side anastomosis. Rings have diameters $1 / 4$ and $3 / 4$ graft diameter respectively. Note strong stretching of outer ring which produces pair of vortices.
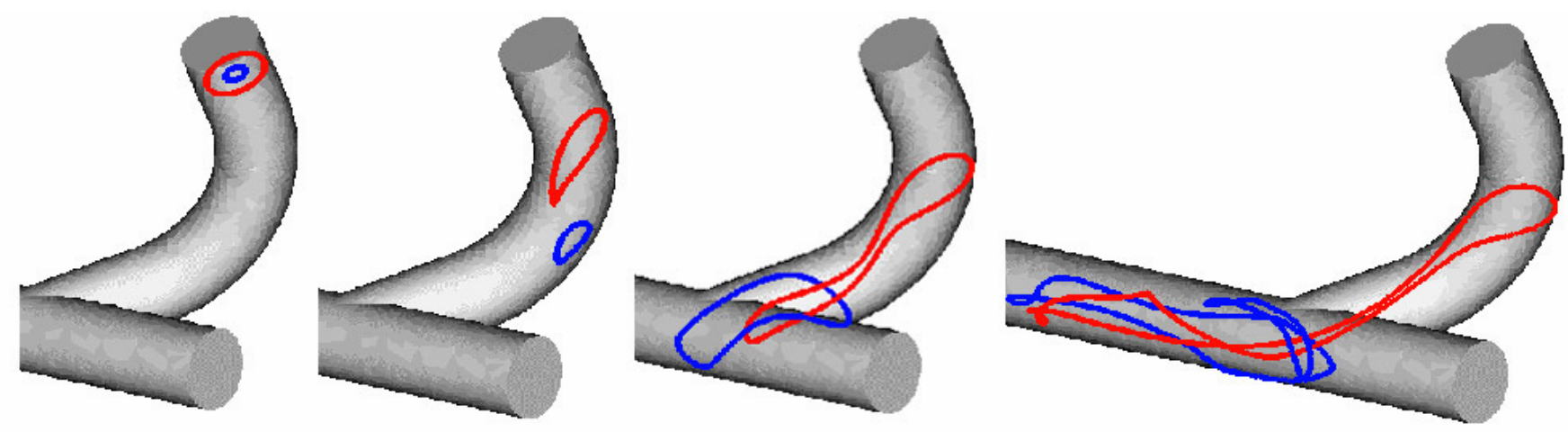

Figure 6

Non-planar graft results for transport of particle rings of $1 / 4$ and $3 / 4$ graft vessel diameter corresponding to preceding results for planar graft. 


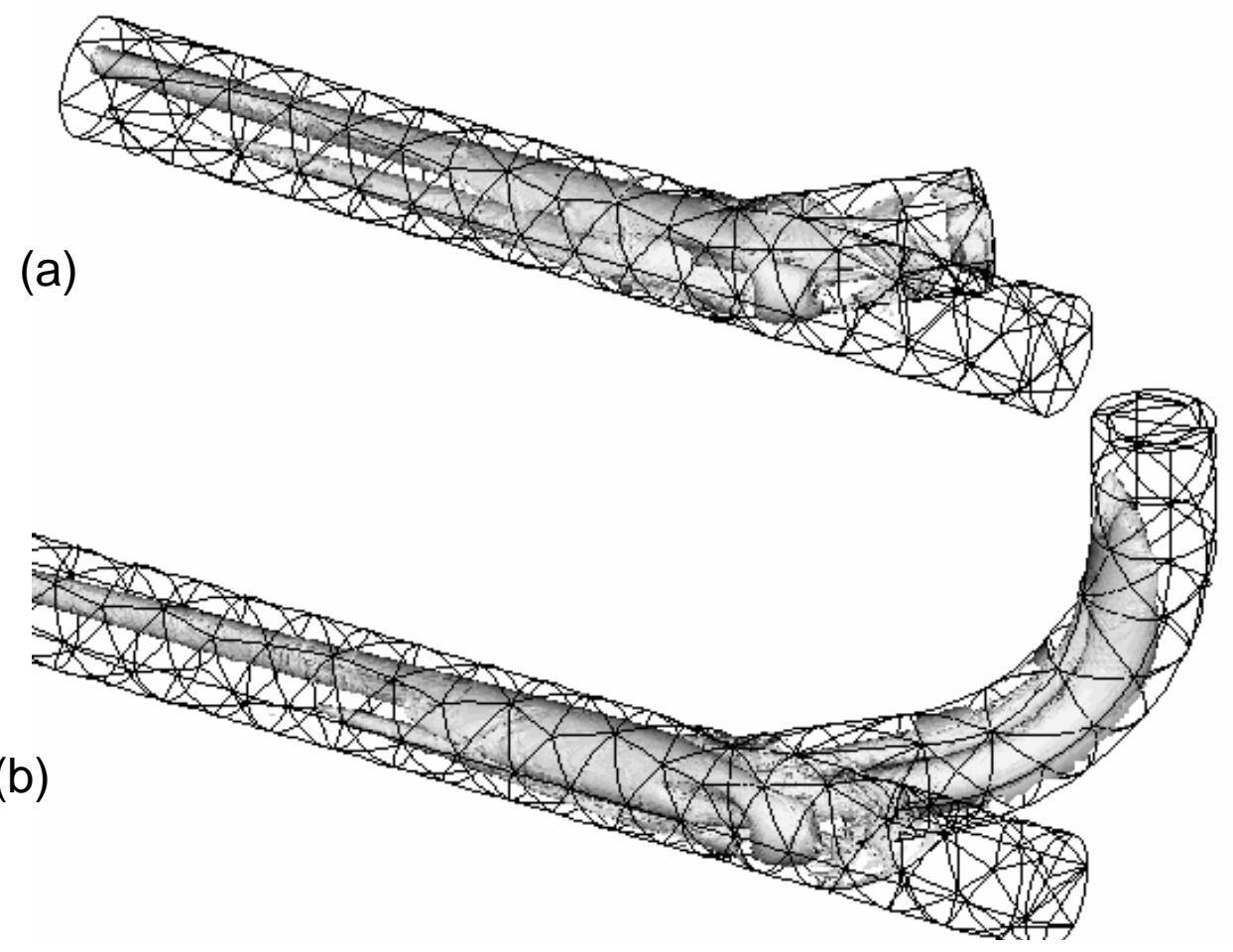

\section{Figure 7}

Comparison of flow structures for:

(a) planar graft with modified inflow (i.e. prescribed to match axial component of non-planar graft)

(b) non-planar graft.

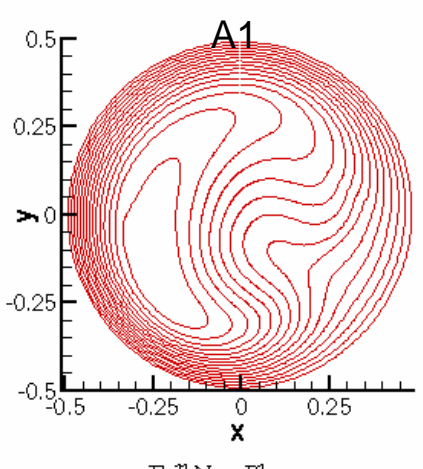

Full Non-Planar

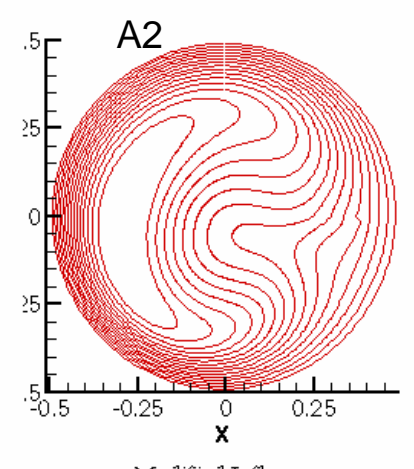

Modified Inflow

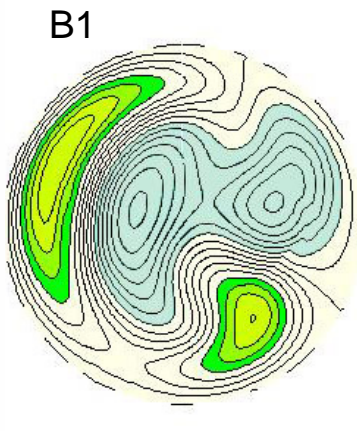

Planar us Non-planar
$[-0.5-0.5]$
B2

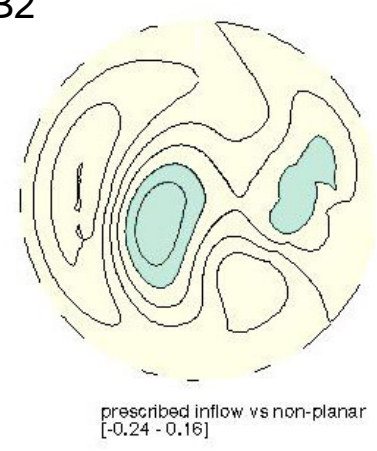

Figure 8A, 8B

Axial flow at 2D downstream of toe.

A1: non-planar, A2 planar modified inflow

Contours of difference in axial flow .

B1: difference $=$ non-planar graft - planar graft

B2: difference $=$ non-planar graft - modified inflow 


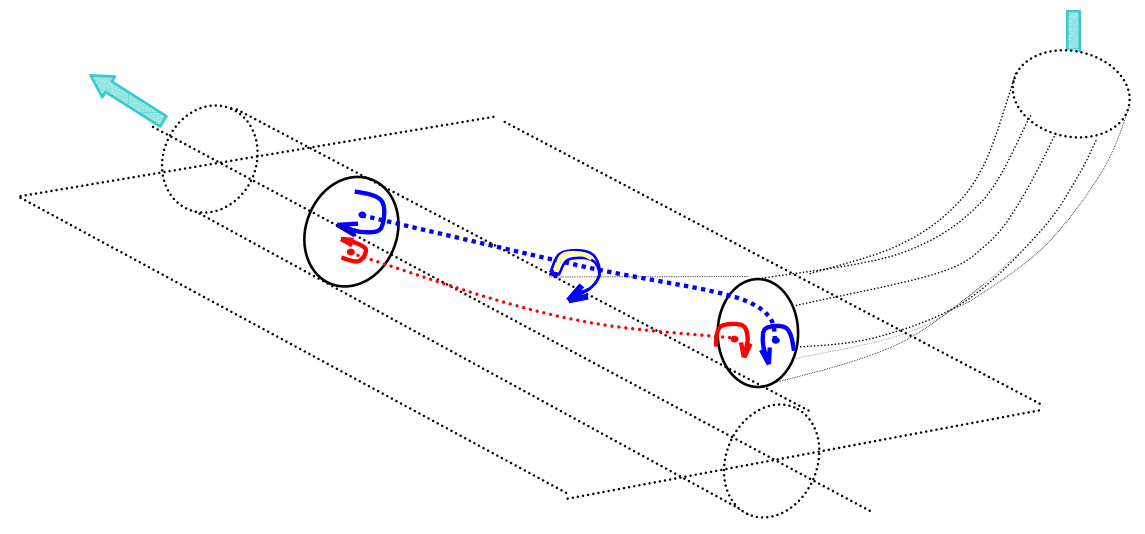

\section{Figure 9}

Sketch indicating directions of rotation of Dean vortices in curved graft, and vortices in host vessel outflow. The clockwise (in flow direction) vortex is reinforced by the flow reorientation in the host, whereas the anti-clockwise vortex is diminished.
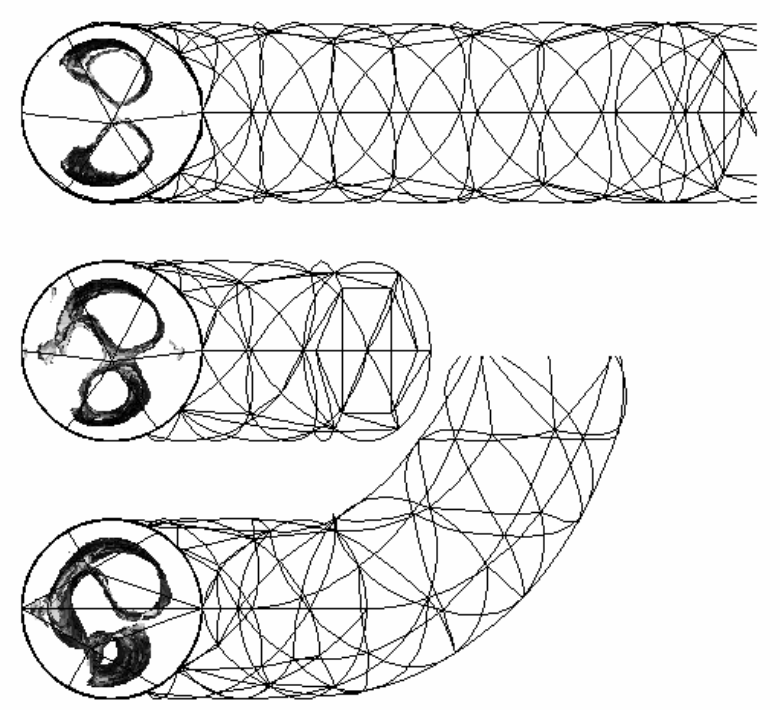

Figure 10

Vortex core locations at 2.5 diameters downstream of toe revealed by removing $\lambda_{2}$ isosurface top: planar graft, middle: planar with modified inflow, bottom: non-planar graft 

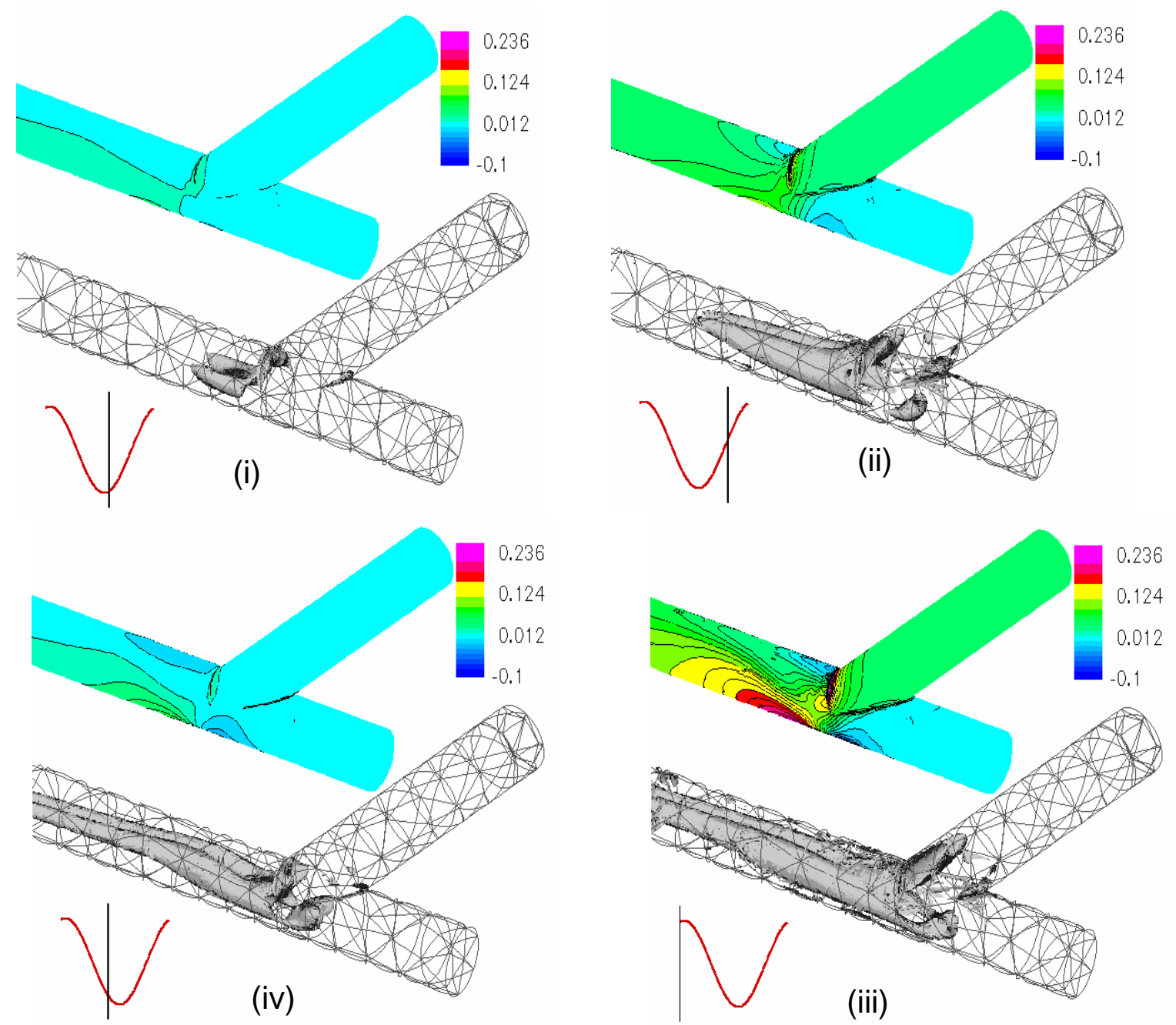

\section{Figure 11}

Flow structures and wall shear in unsteady graft flow

Increasing marker size, and colour change from blue to red indicates increased shear exposure in the plot. 

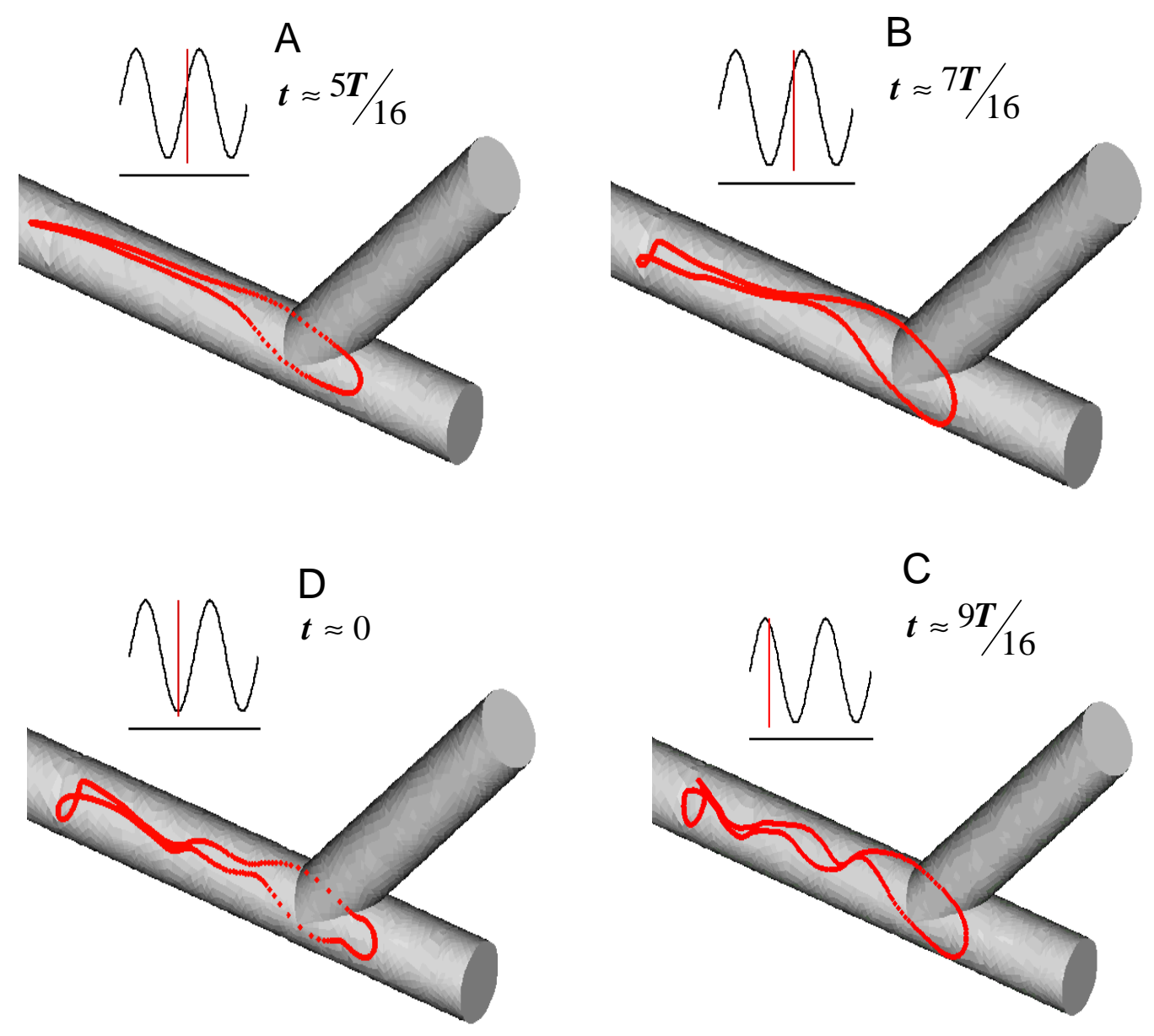

\section{Figure 12}

Positions of marker particle rings released in graft at different instants in unsteady flow. Image time shown, corresponding release times are such that ring enters host at: $\mathrm{A}: t \approx T / 16 ; \mathrm{B}: t \approx T / 4 ; \quad \mathrm{C}: t \approx 7 T / 16 ; \quad \mathrm{D}: t \approx 3 T / 4$ 
A
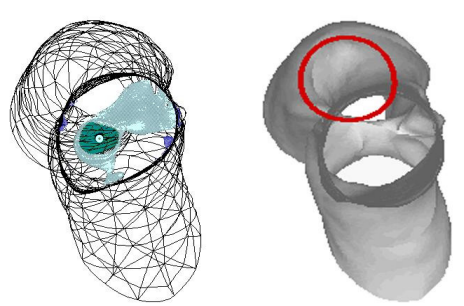

1
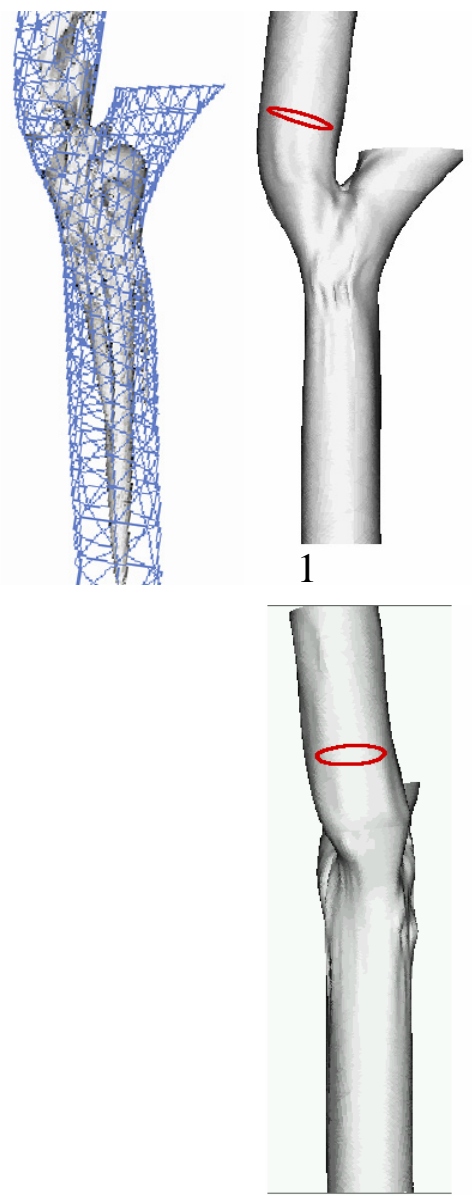

B

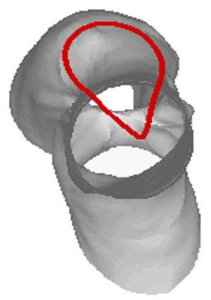

2

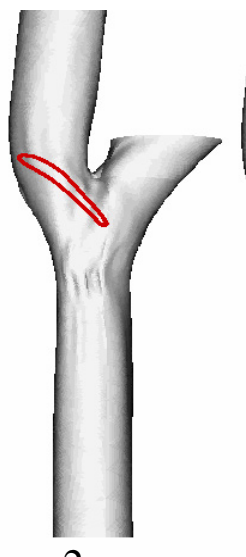

2

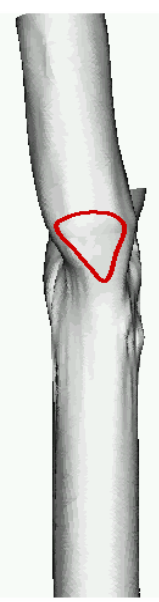

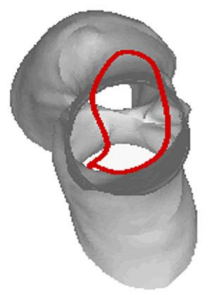

3

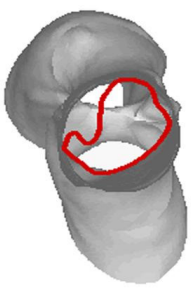

4

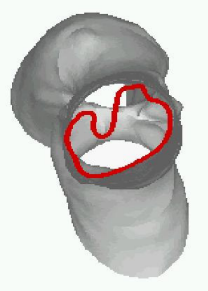

5

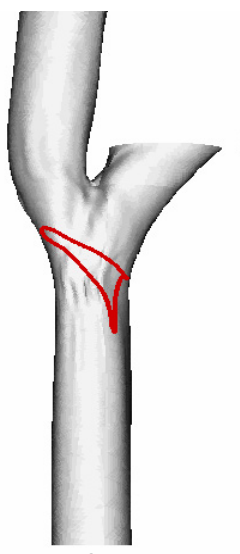

3
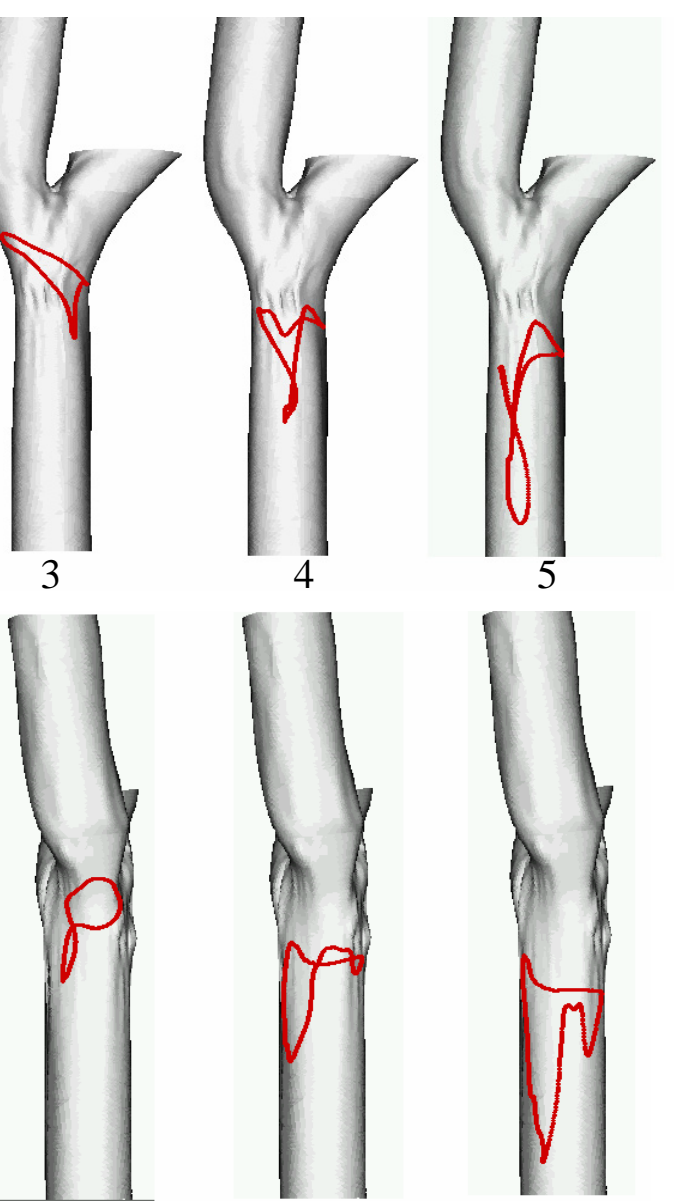

Figure 13

Reconstructed coronary bypass graft: vortical structures and dynamics in steady flow. Host artery outflow is blended to circular tube at 3D downstream of anastomosis, proximal portion is fully occluded.

A: $\quad \lambda_{2}$ isosurface. In upper (head-on) view, portions of the isosurface outside region $1 \frac{1}{2}$ to $2 \frac{1}{2}$ diameters downstream of toe are removed, revealing core of dominant vortex, (bright dot on dark shading).

B: $\quad$ Sequence of images showing position of marker particle ring at equi-spaced intervals labelled $1-5$. Initial ring is $3 / 4$ graft diameter. 

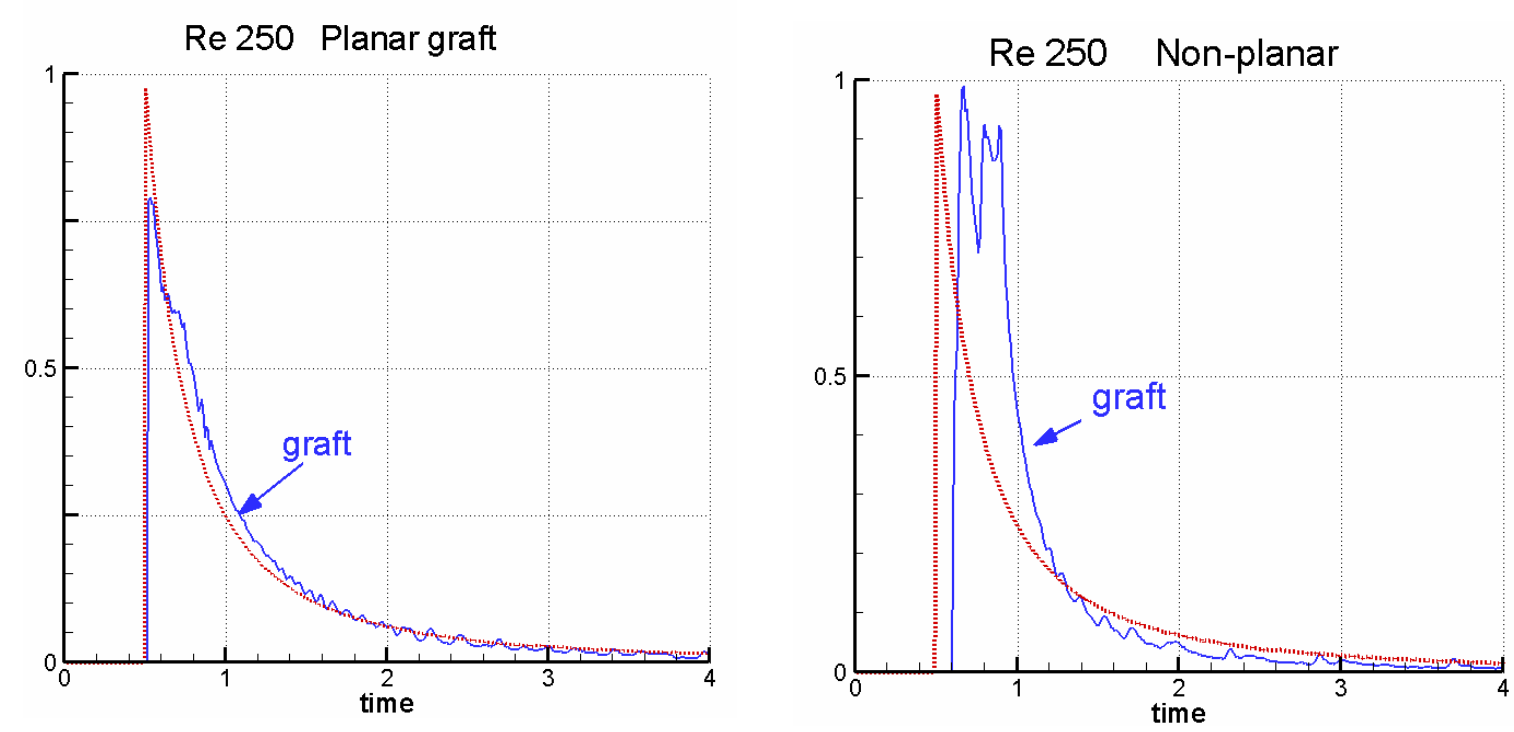

\section{Figure 14}

Exit time distribution at 3D downstream of toe for disk of approx. 70,000 particles uniformly distributed across graft and released

(a) 0.5 diameters upstream of start of bend in graft for non-planar geometry

(b) 2.5 diameters upstream of toe for planar geometry.

Distribution for particles to travel equivalent distance in Poiseuille flow shown for comparison. Time normalised by transit time for median particle in Poiseuille flow.

Planar geometry flow characterised by relatively mild difference in distribution from Poiseuille case.

Non-planar geometry shows both delayed arrival and more compact shape, indicating more uniform velocity distribution and better mixing. 


\section{a: Planar}
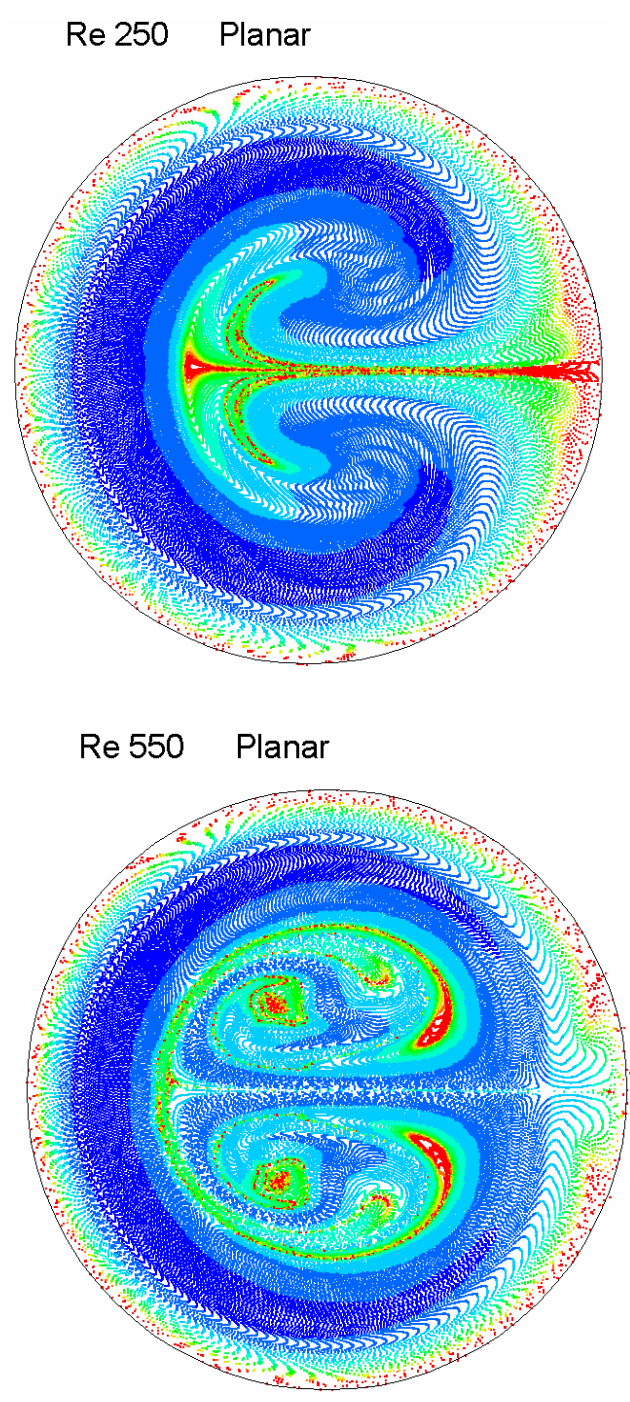

\section{b: Non-planar}
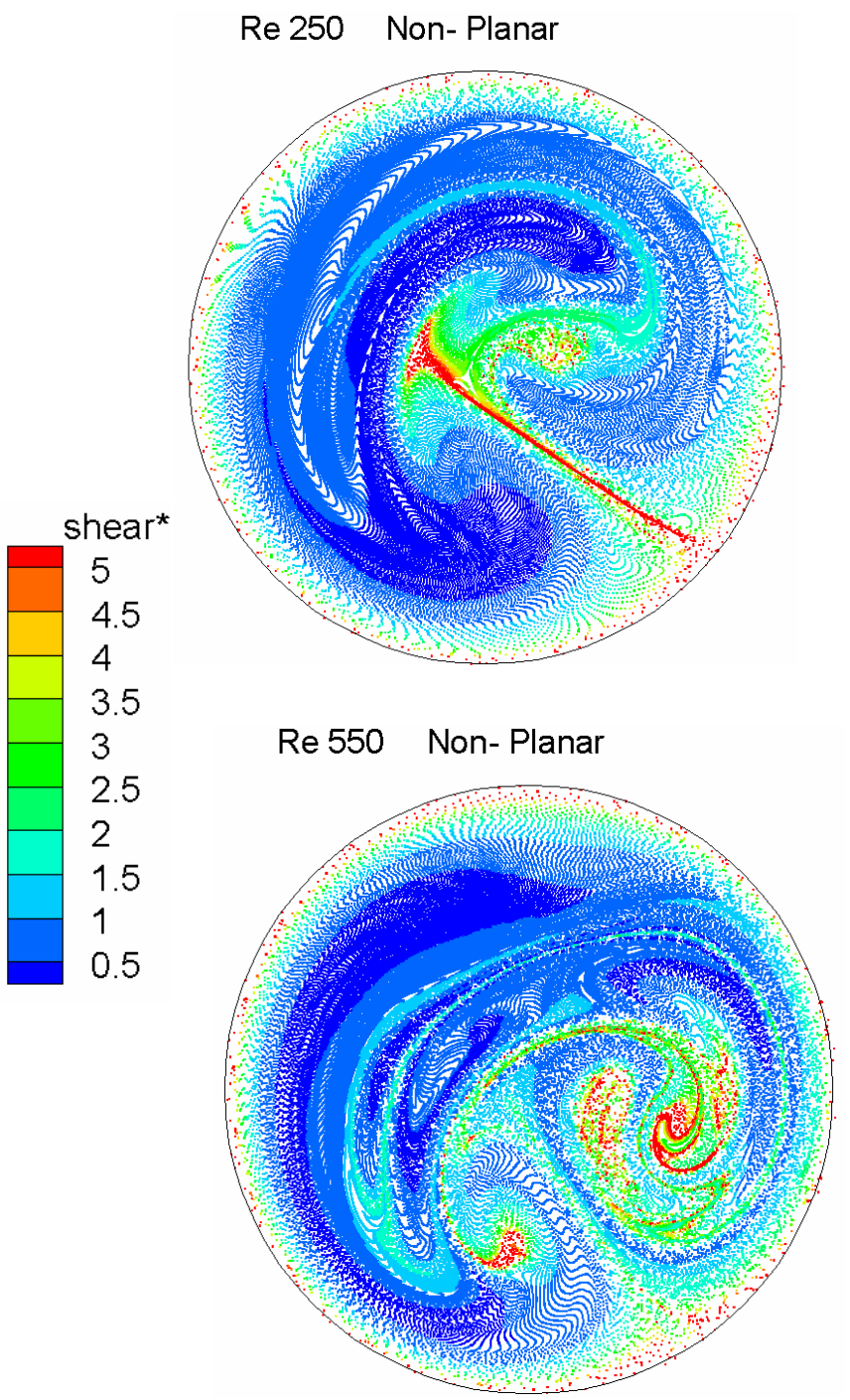

Figure 15

Comparison of distribution of normalised shear loaded particles for planar (left) and nonplanar (right) grafts at 3D downstream of toe in steady flow at Re 250 (top) and Re 550 (bottom). Shear accumulated by a particle at a median radius (i.e. $R / \sqrt{ } 2$ ) in Poiseuille flow used for normalisation.

Particles initially seeded uniformly over the tube cross-section 0.5 diameters upstream of entrance to bend in graft in non-planar case, and at 2.5 diameters from toe in planar case.

Note that as Re increases, the vortices become stronger, and near wall particles which accumulate shear are already swept into core flow by 3D. Also note more complete mixing of shear laden particles for non-planar case, where vortical structure reorientation occurs from graft bend to downstream host artery, and a single vortex dominates artery flow. 
a

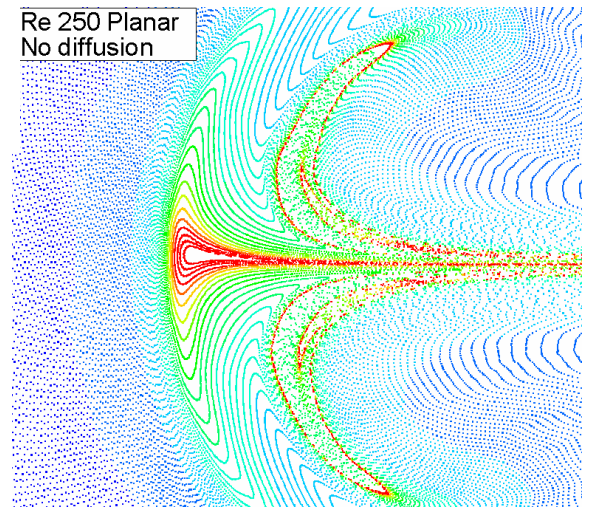

$\mathrm{b}$

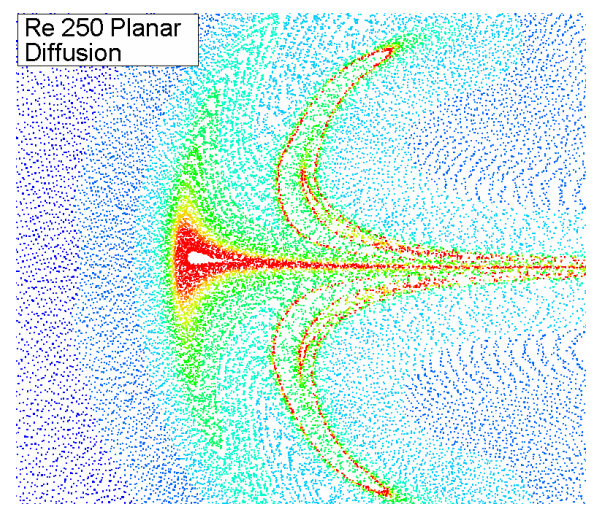

C

d

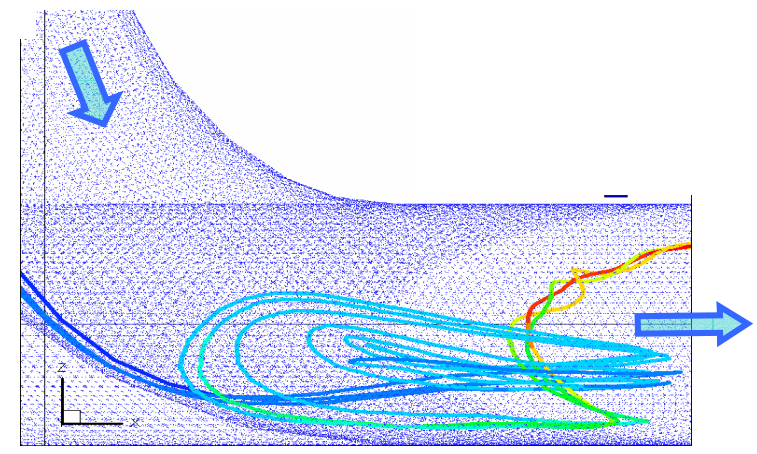

\section{Figure 16}

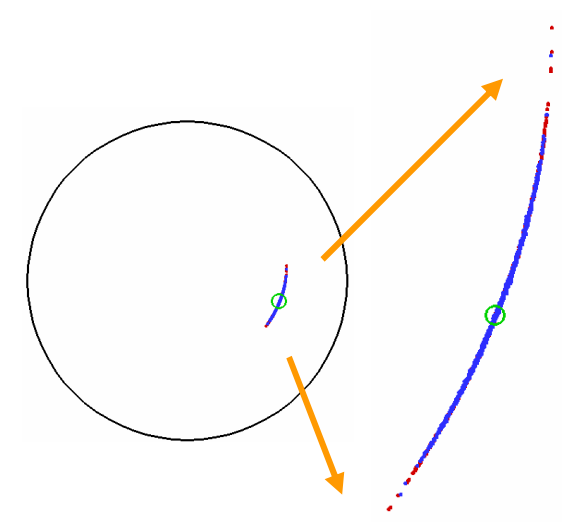

Effects of small diffusion on Lagrangian dynamics in steady flow.

(a) , (b). Fine details of the exit plane shear distribution map at Re 250 in the planar geometry of figure 14, appear similar whether diffusion is present (b) or absent (a).

(c), (d). Wiener bundle of trajectories originating from given initial point in release disk in non-planar flow indicates however that in regions of rapidly varying gradient, the probable shear exposure (c) of a particle, and its spatial localisation at the exit plane (d) may have significant variance.

Colour levels in (a),(b),(c) indicative of accumulated shear; 3 illustrative trajectories shown in c; particle spread in (d) computed for 1,000 particles with constant (blue) and shear-enhanced (red) diffusion. 